\title{
MACrorRestos Vegetales en El Sitio ARqueOlógico Cueva Salamanca 1, Antofagasta de la Sierra (Catamarca, Argentina). Paleoambiente y uso de la VEGETACIÓN DURANTE EL HOLOCENO
}

\author{
Plant macroremains at Cueva Salamanca 1 archaeological \\ site, Antofagasta de la Sierra (Catamarca, Argentine). \\ Paleoenvironment and Vegetation use during the Holocene
}

\author{
Nancy Mariel Apóstolo ${ }^{1 *} \oplus$, María Fernanda Rodríguez ${ }^{2}$ y Elizabeth Pintar $^{3}$
}

\begin{abstract}
Citar este artículo APÓSTOLO, N. M., M. F. RODRÍGUEZ \& E. PINTAR. 2019. Macrorrestos vegetales en el sitio arqueológico Cueva Salamanca 1, Antofagasta de la Sierra (Catamarca, Argentina). Paleoambiente y uso de la vegetación durante el Holoceno. Bol. Soc. Argent. Bot. 54: 43-65.
\end{abstract}

DOI: http://dx.doi. org/10.31055/1851.2372.v54. n1.23586

\section{SUMMARY}

Background and aims: The archaeological site Cueva Salamanca 1 (CS1), Antofagasta de la Sierra (Catamarca, Argentina), is related to the grassland, tolar and lowland plant associations and was inhabited by hunter-gatherer groups (ca. 10,000 6,000 years BP). The plant remains recovered from the CS1 site are studied in order to determine the palaeoenvironmental conditions that occurred during the Holocene, which determined the use and specific exploitation of different plant associations by human groups that inhabited the site.

M\&M: The plant macroremains recovered from CS1 were analysed with microscopy and by morphological comparisons with the species of the current flora surrounding the site.

Results: Of the 60 plant species that grow in the current environment related to the CS1 archaeological site, $44 \%$ are represented in the recovered, both herbaceous and woody charred and non-charred, belonging to the families Asteraceae, Brassicaceae, Cactaceae, Caryophyllaceae, Chenopodiaceae, Ephedraceae, Fabaceae, Malvaceae, Poaceae, Solanaceae and Verbenaceae. A greater use of the tolar is observed, followed by the grassland and the lowland during the whole sequence, considering the species that grow in a radius of $30 \mathrm{~km}$ from the site.

Conclusions: The human groups that inhabited CS1, during the Holocene Early Middle, Late Middle and Late, have made an effective occupation and knew and identified the different plant species to be exploited as fuel, preparation of manufactures and resting litters. The morphological and anatomical adaptations observed in the species found reflect the conditions of the paleoenvironment during the Holocene.

\section{KeY Words}

Archaeobotany, Holocene, hunter-gatherers, paleoenvironment, plant macroremains.

\section{RESUMEN}

Introducción y objetivos: El sitio arqueológico Cueva Salamanca 1 (CS1), Antofagasta de la Sierra (Catamarca, Argentina), está relacionado a las asociaciones vegetales pajonal, tolar y vega y fue habitado por grupos cazadores-recolectores (ca. 10.000 - 6.000 años AP). Se estudian los restos vegetales recuperados del sitio CS1, con el fin de determinar las condiciones paleoambientales que se sucedieron durante el Holoceno, las cuales determinaron el uso y la explotación específica de diferentes asociaciones vegetales por parte de grupos humanos que lo habitaban.

M\&M: Los macrorrestos vegetales recuperados fueron analizados con microscopía y mediante comparaciones morfológicas con las especies de la flora actual circundante al sitio.

Resultados: De las 60 especies vegetales que crecen en el ambiente actual relacionado al sitio CS1, $44 \%$ están representadas en los macrorrestos recuperados, tanto herbáceos como leñosos no carbonizados y carbonizados, pertenecientes a las familias Asteraceae, Brassicaceae, Cactaceae, Caryophyllaceae, Chenopodiaceae, Ephedraceae, Fabaceae, Malvaceae, Poaceae, Solanaceae y Verbenaceae. Se observa un mayor uso del tolar, seguido por el pajonal y la vega durante toda la secuencia, considerando las especies que crecen en un radio de $30 \mathrm{~km}$ a partir del sitio.

Conclusiones: Los grupos humanos que habitaron CS1, durante el Holoceno Medio temprano, Medio tardío y Tardío, han efectuado una ocupación efectiva y conocían e identificaban las distintas especies vegetales para ser explotadas como combustible, confección de tecnofacturas y camadas de descanso. Las adaptaciones morfológicas y anatómicas observadas en las especies halladas reflejan las condiciones del paleoambiente durante el Holoceno.

Palabras clave

Arqueobotánica, cazadores-recolectores, Holoceno, macrorrestos vegetales, paleoambiente. 


\section{INTRODUCCIÓN}

El sitio arqueológico Cueva Salamanca 1 (CS1) se encuentra ubicado en la microrregión de Antofagasta de la Sierra (ANS), Catamarca, Puna Sur de la Argentina que, de acuerdo con Troll (1958), corresponde a la Puna Salada. El área puede describirse como un ambiente semidesértico e integra la Región Puneña en donde la vegetación predominante corresponde a la estepa arbustiva, desértica y semidesértica (Cabrera, 1976) (Fig. 1A-B).

De acuerdo con los estudios arqueobotánicos realizados hasta la fecha, durante los primeros 4.000 años del Holoceno, ca. 10.000 - 6.000 años AP, los grupos nómades que habitaron la microrregión de ANS basaron su economía en actividades extractivas de caza-recolección. A partir de $c a$. 6.000 años AP se habría dado un pasaje paulatino hacia economías productivas que incorporaron una agricultura incipiente junto con el pastoralismo, dando lugar a un mayor sedentarismo. No obstante, continuaron practicando actividades de caza y recolección durante un largo periodo.

Sobre la base de la composición florística y la cobertura vegetal, se definieron en ANS tres asociaciones vegetales: pajonal, tolar y vega (Fig.1D-F). Por encima de los $3.800 \mathrm{~m}$ s. m., se desarrolla un pastizal de gramíneas tales como Festuca sp., Stipa sp. y Deyeuxia sp., con especies subarbustivas de los géneros Adesmia (Fabaceae), Baccharis (Asteraceae), Parastrephia (Asteraceae) y Fabiana (Solanaceae); éste corresponde a la asociación vegetal del pajonal. Por debajo de los $3.800 \mathrm{~m} \mathrm{~s}$. m. comienza el tolar, en donde abundan especies de Parastrephia (Asteraceae) y Acantholippia (Verbenaceae). El cauce del Río Las Pitas se caracteriza por presentar una cubierta de gramíneas y juncáceas que conforman la vega (Rodríguez, 2000; Rodríguez et al., 2006).

En relación con el paleoambiente, sobre la base de estudios sedimentológicos, granulométricos, de diatomeas (Tchilinguirian, 2008; Tchilinguirian \& Olivera, 2005; Tchilinguirian et al., 2008; Tchilinguirian \& Morales, 2013) y florísticos en general en la Puna Norte y Sur (Markgraf \& Bradbury, 1982; Markgraf, 1985, 1987; Fernández et al., 1991; Lupo \& Echenique, 2001), se plantea la siguiente secuencia: durante el Holoceno Temprano $(10.000-8.500$ AP) el clima habría sido más frío y más húmedo que el actual, mientras que durante el Holoceno Medio (8.500 - 3.600 AP) se registraría un clima más cálido y más seco; el Holoceno Tardío (3.600 AP en adelante) marca el comienzo de las condiciones climáticas semejantes a las actuales.

Para el Holoceno Temprano (10.000-8.500 AP) con clima más húmedo y menos estacional, se presume que los grupos cazadores-recolectores ingresaron en el área, se dispersaron explorando distintos micro-ambientes, ocupando sitios durante breves lapsos lo cual supone una elevada movilidad residencial (Franco, 2002; Civalero \& Franco, 2003). A medida que el proceso de colonización continuó las ocupaciones habrían sido recurrentes con pequeños hiatos. Sin embargo, alrededor de $c a$. 8.500 AP con el comienzo de condiciones más áridas y un ambiente más estacional, es probable que haya habido un incremento en el tamaño del rango anual de los grupos para evitar la sobre explotación de recursos y el uso de viajes logísticos para obtener materias primas de excelente calidad y también para monitorear áreas (Pintar et al., 2016).

A pesar de los cambios planteados, en la Puna existen áreas con alta concentración de recursos y, por este motivo, es probable que la localización de las comunidades vegetales se haya mantenido más o menos estable a través del tiempo en relación con la altitud, variando únicamente en cantidad y extensión de acuerdo con los cambios paleoambientales, lo cual ofrecería una ventaja al evaluar la vegetación actual y aquella que predominó en el pasado. Es importante tener en cuenta que los trabajos arqueobotánicos en sitios arqueológicos de ANS que forman parte de sistemas de subsistencia-asentamiento, apoyan y refuerzan este planteo (Rodríguez, 1998, 1999, 2000, 2004 a, b, 2005, 2013, 2014; Rodríguez \& Rúgolo de Agrasar, 1999; Rodríguez et al., 2003; Rodríguez \& Aschero, 2005). Estos estudios fueron complementados con aquellos referidos a la flora actual y la información etnobotánica (Haber, 1992; Rodríguez, 1998; Cuello, 2006). Diversos estudios, arqueológicos y etnográficos, acerca de grupos cazadores-recolectores indican que éstos eligen puntos centrales dentro de sus sistemas de asentamiento desde donde organizan partidas de caza y recolección para explotar los recursos y maximizar el rendimiento del forrajeo. Los análisis acerca del uso de recursos líticos y vegetales, sobre la base de la procedencia de las materias primas utilizadas en varios sitios de ANS, sugieren que 

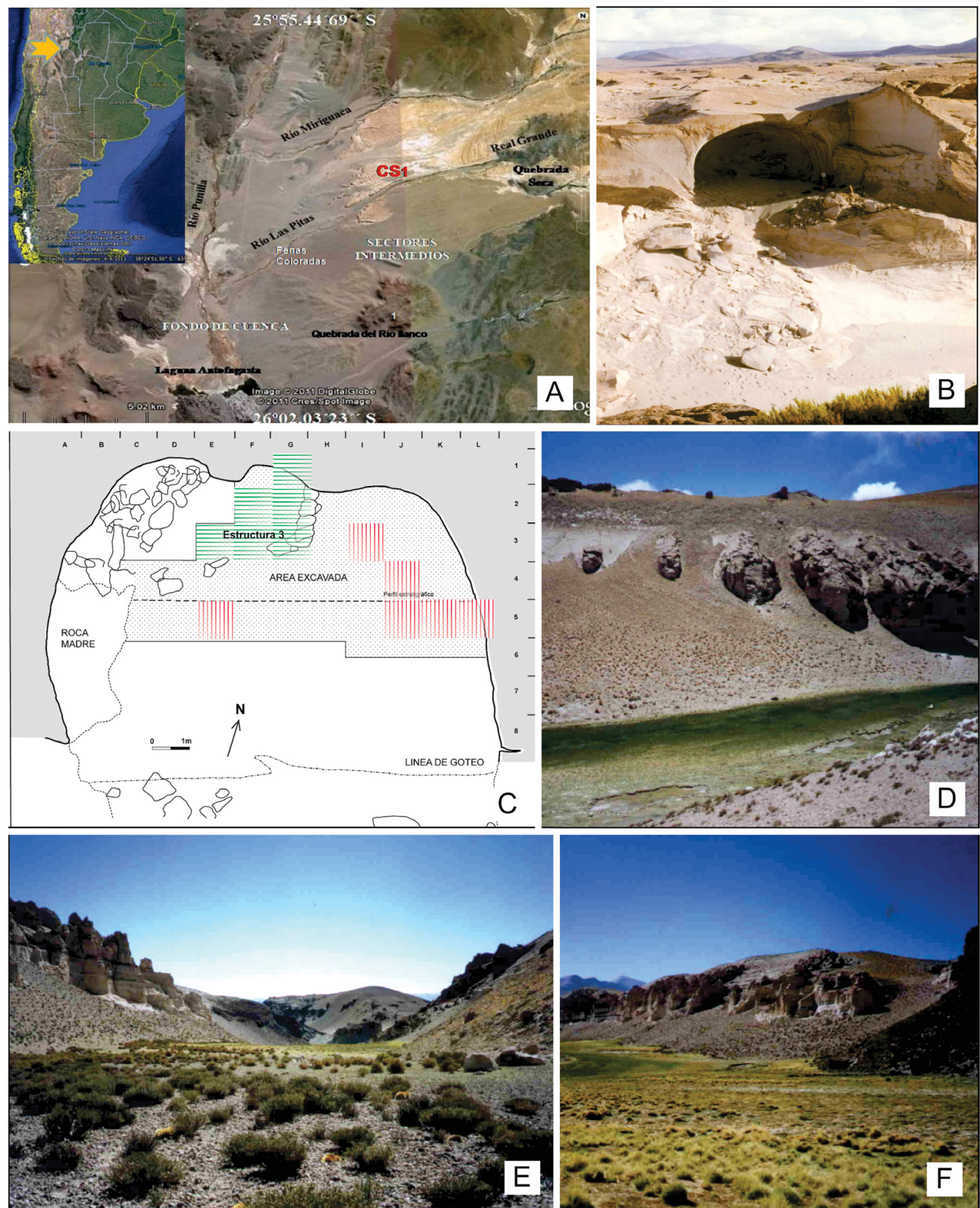

Fig. 1. Sitio Cueva Salamanca 1, Antofagasta de la Sierra (Catamarca, Argentina). A: Ubicación geográfica. B: Vista del sitio. C: Planta y cuadrículas de excavación en el sitio. D-F: Tipo de vegetación asociada al sitio en la actualidad. D: Vega. E: Tolar. F: Pajonal. 
el radio de forrajeo era aproximadamente de 2-3 $\mathrm{km}$ alrededor de las bases residenciales durante el Holoceno Temprano mientras que estos radios se expandieron a 6-7 $\mathrm{km}$ durante el Holoceno Medio (Grove, 2009, 2010; Kelly, 2013; Pintar \& Rodríguez, 2015).

Dado que las especies vegetales recuperadas en el sitio CS1 fueron utilizadas por los distintos grupos humanos que lo habitaron y que, por otra parte, la presencia de muchos taxones en el sitio obedece a causas naturales, el análisis del registro arqueobotánico brindaría información paleoambiental a través del estudio de las adaptaciones morfológicas y anatómicas de las especies halladas. Por ello, se plantea la hipótesis de que las condiciones paleoambientales que se sucedieron durante el Holoceno determinaron el uso y la explotación específica de diferentes asociaciones vegetales de donde proceden los taxones hallados en el registro arqueobotánico de CS1.

A partir de estas consideraciones, el objetivo general de este trabajo es contrastar las características adaptativas morfológicas y anatómicas de los macrorrestos vegetales recuperados en el sitio CS1 con los modelos paleoambientales producidos hasta el momento sobre la base de datos no botánicos, estableciendo los cambios y las continuidades en el manejo del medio ambiente natural y, más específicamente, en el uso de los recursos vegetales durante distintos momentos del Holoceno. De este modo, sería posible contrastar el ambiente y el paleoambiente a través de la vegetación e indagar acerca del uso de las plantas tanto en el presente como en el pasado prehispánico.

\section{Materiales y Métodos}

\section{Sitio arqueológico}

El sitio CS1 se encuentra en el sector del curso medio-inferior del Río Las Pitas -localidad arqueológica Punta de la Peña- a 3.665 m s. m., en la asociación vegetal que corresponde al tolar y sus coordenadas son $26^{\circ} 01^{\prime} 22^{\prime}$ ' S y $67^{\circ} 15^{\prime} 23^{\prime \prime} \mathrm{W}$ (Fig. 1 A, B, D). La cueva yace en una cañada transversal a la Quebrada del Río Las Pitas, dentro de las peñas de ignimbritas que bordean la terraza del río. Está orientada al sur y sus dimensiones son $11 \mathrm{~m}$ de ancho, $8 \mathrm{~m}$ de profundidad y $7 \mathrm{~m}$ de alto. El área de reparo dentro del sitio es de aproximadamente $77 \mathrm{~m}^{2}$. La topografía es muy irregular con escasa cobertura vegetal y, de acuerdo con su posición, controla la única cañada de acceso al relieve que corresponde a una meseta de ignimbritas que rodea la cuenca del Río Las Pitas (Pintar, 2004) (Fig. 1B).

La secuencia arqueológica general del sitio fue definida en 3 capas o unidades estratigráficas. En la capa 1 con cerámica - ca.1.800 AP- se definieron dos ocupaciones denominadas 1(1) y 1(2), seguidas por una lente de ceniza volcánica estéril. En la capa 2, correspondiente a momentos pre-cerámicos, hubo 10 ocupaciones comprendidas entre $c a$. 4.500-8.100 años AP: 2(1) a 2(10). Por debajo de ésta, hay una capa de arena estéril que apoya sobre la roca madre. Los hallazgos botánicos, líticos y faunísticos indican que este sitio fue una base residencial de grupos cazadores-recolectores durante un período comprendido entre $c a$. 6.200 - 8.100 años AP (Holoceno Medio temprano y comienzos del Holoceno Medio tardío) (Tabla 1).

Por otra parte, en CS1 se definió un sector al que se denominó estructura 3 que apoya sobre la pared de la cueva (Fig. 1C). En la misma se diferenciaron cinco extracciones que se corresponden con las capas 1(1), 1(2), 2(1), 2(2) y 2(3) respectivamente, alcanzando luego la roca madre, en las cuales se recuperaron haces de gramíneas con o sin estructuras reproductivas, restos de plantas (flores, hojas, semillas y frutos), macrorrestos leñosos, así como también huesos de animales, material lítico y cordeles (Pintar, 1996, 2004; Rodríguez, 2005).

\section{Análisis cualitativo de muestras y datos}

Se realizó un muestreo de los macrorrestos vegetales (herbáceos y leñosos) recuperados en las capas 1 y 2 de la estructura 3 (cuadrículas E3, F2, F3, G1, G2 y G3), y en el sector de la cueva opuesto a esta estructura en las cuadrículas J4, J5, K5, L5, I3 y E4 (esta última es la más cercana a la mencionada estructura) (Fig. 1C). Específicamente en la capa 2 de este último sector, por fuera de la estructura 3 , se analizaron los macrorrestos hallados en 2(1) a 2(8) ya que en 2(9) y 2(10) no se registró la presencia de restos botánicos para las mencionadas cuadrículas. Estos materiales fueron registrados en su totalidad y caracterizados macroscópicamente, para luego ser identificados mediante estudios anatómicos y/o morfológicos comparativos a partir del material actual coleccionado en el área de 


\section{N. M. Apóstolo et al. - Macrorrestos vegetales en el sitio arqueológico Cueva Salamanca 1}

\section{Tabla 1. Secuencia estratigráfica del sitio Cueva Salamanca 1, Antofagasta de la Sierra (Catamarca, \\ Argentina). Dataciones radiocarbónicas de las capas 1 y 2. Abreviaturas: HMtem, Holoceno Medio temprano; HMtardío, Holoceno Medio tardío; HTardío, Holoceno Tardío.}

\begin{tabular}{|ccccc|}
\hline Capa & $\begin{array}{c}\text { Fechado convencional } \\
\text { (años AP - C14) } \\
\text { Posterior a 1796 }\end{array}$ & Material & Laboratorio & Período \\
\hline $1(1)$ & $1796 \pm 23$ & Carbón & AA107153 & HTardío \\
$1(2)$ & $4460 \pm 30$ & Carbón & Beta-311872 & HMtardío \\
\hline $2(1)$ & $6250 \pm 70$ & Carbón & LP-931 & HMtardío \\
$2(2)$ & $7410 \pm 100$ & Carbón & Beta-311873 & HMtem \\
$2(3)$ & $7630 \pm 40$ & Carbón & LP-615 & HMtem \\
& $7500 \pm 60$ & Carbón & Beta-178223 & HMtem \\
$2(4)$ & $7550 \pm 60$ & Carbón & Beta-178224 & HMtem \\
$2(5)$ & $7540 \pm 50$ & Carbón & Beta-280987 & HMtem \\
$2(6)$ & $7620 \pm 60$ & Carbón & Beta-178225 & HMtem \\
$2(7)$ & $7870 \pm 50$ & Carbón & Beta-280988 & HMtem \\
$2(8)$ & $7990 \pm 60$ & Carbón & Beta-280989 & HMtem \\
$2(9)$ & $8100 \pm 50$ & Carbón & Beta-260687 & HMtem \\
\hline $2(10)$ & & & & \\
\hline
\end{tabular}

estudio (Rodríguez, 1998) (Tabla 2) y material de herbario pre-existente. Para esto, se confeccionó una histoteca de referencia con preparados histológicos de cortes transversales de tallos y raíces leñosas, cañas y hojas de especies actuales presentes según método convencional (D'Ambrogio de Argüeso, 1986; Zarlavsky, 2014).

Los restos herbáceos recuperados consistieron en estructuras completas o parciales de frutos, semillas, estructuras reproductivas, hojas y tallos herbáceos. Por otra parte, los restos leñosos se encontraron carbonizados (carbón) y no carbonizados (leña en zonas de combustión). Estos macrorrestos fueron individualizados teniendo en cuenta la capa en donde fueron hallados. Todos los materiales recuperados fueron observados y fotografiados con microscopio estereoscópico Zeiss STEMI 2000C.

Macrorrestos leñosos no carbonizados. Se tomaron pequeñas porciones de material y se ablandaron mediante ebullición en agua con unas gotas de lavandina de uso comercial. Esto permitió realizar cortes en forma transversal y longitudinal (radial y tangencial) en secciones de $15-30 \mu \mathrm{m}$ de espesor, usando micrótomo de deslizamiento Micron HM 400R. Dichos cortes fueron coloreados con doble coloración (safranina fast-green) (D'Ambrogio de Argüeso, 1986; Zarlavsky, 2014) y montados en resina sintética para ser observados y fotografiados con cámara adosada al microscopio óptico (MO) Nikon Eclipse E200. También, los cortes transversales y longitudinales gruesos pudieron ser observados y fotografiados con Microscopio Electrónico de Barrido (MEB) (Phillips XL-30 SEM, Servicio de Microscopía del Museo Argentino de Ciencias Naturales Bernardino Rivadavia, CABA).

Macrorrestos leñosos carbonizados. Se efectuaron cortes transversales de trozos de carbón con hojas de afeitar bajo microscopio estereoscópico Zeiss STEMI 2000C. Posteriormente, se observaron $\mathrm{y}$ fotografiaron con MEB.

Macrorrestos herbáceos. Estos materiales fueron observados y analizados morfológicamente por comparación con material actual de referencia $\mathrm{y}$, finalmente, observados y fotografiados con MEB. Además, para analizar las hojas y las cañas floríferas de especímenes de la familia Poaceae se realizaron cortes transversales a mano alzada de la lámina de la segunda hoja caulinar en su parte 


\section{Bol. Soc. Argent. Bot. 54 (1) 2019}

Tabla 2. Especies presentes en el ambiente actual asociado al sitio arqueológico Cueva Salamanca 1 , Antofagasta de la Sierra (Catamarca, Argentina). Relación con la asociación de vegetación, altitud y uso actual.

\begin{tabular}{|c|c|c|c|c|c|}
\hline Familia & Nombre científico & $\begin{array}{c}\text { Nombre } \\
\text { vernáculo }\end{array}$ & $\begin{array}{c}\text { Asociación } \\
\text { vegetación / altitud }\end{array}$ & $\begin{array}{l}\text { Ejemplar de } \\
\text { referencia }\end{array}$ & Uso actual \\
\hline \multirow[t]{11}{*}{ Asteraceae } & Artemisia copa Phil. & Copa-copa & $\begin{array}{l}\text { Tolar superior y } \\
\text { pajonal inferior / } \\
3700-4000 \text { m s .m. }\end{array}$ & $\begin{array}{l}\text { Rodríguez s.n. } \\
\text { (SI 28.330) }\end{array}$ & $\begin{array}{l}\text { Medicinal: } \\
\text { digestiva }\end{array}$ \\
\hline & $\begin{array}{l}\text { Baccharis salicifolia } \\
\text { (Ruiz \& Pav.) Pers. }\end{array}$ & Suncho & Tolar/3400-3900 m s .m. & $\begin{array}{l}\text { Rodríguez } \\
\text { s.n. (SI) }\end{array}$ & Techos \\
\hline & B. tola Phil. & Lejía & $\begin{array}{c}\text { Laderas de la } \\
\text { Quebrada/>4000 } \\
\text { m s.m. }\end{array}$ & $\begin{array}{l}\text { Rodríguez } \\
18(\mathrm{SI})\end{array}$ & $\begin{array}{l}\text { Combustible, } \\
\text { medicinal }\end{array}$ \\
\hline & $\begin{array}{l}\text { Cotula mexicana } \\
\text { (DC.) Cabrera }\end{array}$ & & Tolar/3400-3900 m s. m. & SI 28.222 & Sin datos \\
\hline & $\begin{array}{c}\text { Chuquiraga } \\
\text { atacamensis Kuntze }\end{array}$ & $\begin{array}{l}\text { Monte } \\
\text { de suri }\end{array}$ & Tolar/3400-3900 m s. m. & SI 28.205 & Sin datos \\
\hline & $\begin{array}{c}\text { Ocyroe armata } \\
\text { (Webb.) Bonifacino } \\
\text { (=Nardophyllum } \\
\text { armata) }\end{array}$ & Suri-yanta & Tolar/3400-3900 m s. m. & $\begin{array}{l}\text { Rodríguez } \\
3(\mathrm{SI})\end{array}$ & Sin datos \\
\hline & $\begin{array}{l}\text { Parastrephia lucida } \\
\text { (Meyen) Cabrera }\end{array}$ & Tola de río & $\begin{array}{l}\text { Vega /3300 - } \\
3400 \text { m s. m. }\end{array}$ & $\begin{array}{l}\text { Rodríguez } \\
11(\mathrm{SI})\end{array}$ & Combustible \\
\hline & $\begin{array}{l}\text { P. quadrangularis } \\
\text { (Meyen) Cabrera }\end{array}$ & Chacha tola & $\begin{array}{l}\text { Laderas de la } \\
\text { Quebrada/4000-4700 } \\
\text { m s.m. }\end{array}$ & SI 28.325 & $\begin{array}{c}\text { Combustible, } \\
\text { sahumador, } \\
\text { delimita corrales }\end{array}$ \\
\hline & Senecio puchii Phil. & & Tolar/3400-3900 m s. m. & SI 28.326 & $\begin{array}{l}\text { Medicinal: } \\
\text { digestiva }\end{array}$ \\
\hline & S. santelicis Phil. & Mocoraca & Tolar/3400-3900 m s. m. & $\begin{array}{l}\text { Rodríguez } \\
2(\mathrm{SI})\end{array}$ & Combustible \\
\hline & $\begin{array}{l}\text { S. xerophilus Phil. } \\
\text { var. xerophilus }\end{array}$ & Sin datos & Tolar/3400-3900 m s. m. & $\begin{array}{l}\text { SI } 28.204, \\
\text { Rodríguez } \\
16(\mathrm{SI})\end{array}$ & Sin datos \\
\hline Boraginaceae & Cryptantha sp. & Sin datos & Tolar/3400-3900 m s. m. & SI 28.216 & Sin datos \\
\hline Brassicaceae & $\begin{array}{l}\text { Neuontobotrys } \\
\text { tarapacanus (Phil.) } \\
\text { Al-Shebahz. }\end{array}$ & Chuchar & Tolar/3400-3900 m s. m. & SI 28.208 & Forraje \\
\hline \multirow[t]{2}{*}{ Cactaceae } & $\begin{array}{l}\text { Maihuenopsis } \\
\text { glomerata (Haw.) } \\
\text { R. Kiesling. }\end{array}$ & Copana & $\begin{array}{c}\text { Tolar/3400 }-3900 \\
\text { m s. m. }\end{array}$ & SI s.n. & Sin datos \\
\hline & $\begin{array}{l}\text { Tunilla tilcarensis } \\
\text { (Backed.) D:R. } \\
\text { hunt \& Iliff. (T. cfr. } \\
\text { Soehrensii) }\end{array}$ & Sin datos & Tolar/3400-3900 m s. m. & $\begin{array}{l}\text { Rodríguez } \\
23(\mathrm{SI})\end{array}$ & Sin datos \\
\hline Caryophyllaceae & $\begin{array}{l}\text { Pycnophyllum } \\
\text { molle Remy. }\end{array}$ & Yareta & Tolar/3400-3900 m s. m. & SI 28.327 & Combustible \\
\hline \multirow[t]{2}{*}{ Chenopodiaceae } & $\begin{array}{l}\text { Atriplex imbricata } \\
\text { D. Dietr. }\end{array}$ & Cachiyuyo & Tolar/3400-3900 m s. m. & SI 28.212 & $\begin{array}{c}\text { Forraje (Haber, } \\
\text { 1987) }\end{array}$ \\
\hline & $\begin{array}{l}\text { Chenopodium quinoa } \\
\text { Willd.var. quinoa }\end{array}$ & $\begin{array}{l}\text { Quinoa } \\
\text { blanca }\end{array}$ & Tolar/3400-3900 m s. m. & $\begin{array}{l}\text { Aguirre } \\
\text { s.n. (LIL) }\end{array}$ & Comestible \\
\hline Cyperaceae & $\begin{array}{l}\text { Scirpus asper J. } \\
\text { Presl \& C. Presl. }\end{array}$ & Sin datos & $\begin{array}{l}\text { Vega/3300- } \\
3400 \text { m s. m. }\end{array}$ & $\begin{array}{l}\text { Rodríguez } \\
\text { s.n. (SI) }\end{array}$ & Sin datos \\
\hline \multirow[t]{2}{*}{ Ephedraceae } & Ephedra sp. & Tramontana & Tolar/3400-3900 m s. m. & SI 28.214 & Sin datos \\
\hline & $\begin{array}{l}\text { E. multiflora } \\
\text { Phil. ex Stapf }\end{array}$ & Tramontana & Tolar/3400-3900 m s. m. & $\begin{array}{l}\text { Rodríguez } \\
22(\mathrm{SI})\end{array}$ & Combustible \\
\hline
\end{tabular}




\begin{tabular}{|c|c|c|c|c|c|}
\hline Familia & Nombre científico & $\begin{array}{l}\text { Nombre } \\
\text { vernáculo }\end{array}$ & $\begin{array}{c}\text { Asociación } \\
\text { vegetación / altitud }\end{array}$ & $\begin{array}{l}\text { Ejemplar de } \\
\text { referencia }\end{array}$ & Uso actual \\
\hline \multirow[t]{3}{*}{ Fabaceae } & $\begin{array}{l}\text { Adesmia echinus } \\
\text { C. Presl. }\end{array}$ & $\begin{array}{l}\text { Añawa } \\
\text { negra }\end{array}$ & $\begin{array}{c}\text { Laderas de la } \\
\text { Quebrada/4000-4700 } \\
\text { m s.m. }\end{array}$ & SI 28.287 & Combustible \\
\hline & A. horrida Gillies & Añawa & $\begin{array}{c}\text { Tolar superior y } \\
\text { pajonal inferior / } \\
3700-4000 \mathrm{~m} \mathrm{~s} . \mathrm{m} \text {. }\end{array}$ & $\begin{array}{l}\text { SI 28.288, } \\
\text { Rodríguez } \\
13(\mathrm{SI})\end{array}$ & $\begin{array}{l}\text { Combustible, } \\
\text { forrajera }\end{array}$ \\
\hline & $\begin{array}{l}\text { Hoffmanseggia } \\
\text { eremophila (Phil.) } \\
\text { Burkart ex Ulibarri. }\end{array}$ & Algarrobita & Tolar/3400-3900 m s. m. & SI 28.221 & $\begin{array}{c}\text { Comestible } \\
\text { (Ulibarri, 1979) }\end{array}$ \\
\hline Juncaceae & $\begin{array}{l}\text { Juncus cfr. balticus } \\
\text { subsp. mexicanus } \\
\text { Willd. ex Roem. \& } \\
\text { Schult.) Kirschner. }\end{array}$ & Unquillo & $\begin{array}{l}\text { Vega/3300- } \\
3400 \mathrm{~m} \mathrm{s.} \mathrm{m.}\end{array}$ & $\begin{array}{l}\text { Rodríguez } \\
19,24 \text { (SI) }\end{array}$ & Forraje \\
\hline Loasaceae & $\begin{array}{l}\text { Caiophora coronata } \\
\text { (Gillies ex Arn.) } \\
\text { Hook. \& Arn. }\end{array}$ & Rupachico & Tolar/3400-3900 m s. m. & SI 28.219 & Sin datos \\
\hline Malvaceae & Tarasa sp. & Sin datos & Tolar/3400-3900 m s. m. & SI 28.220 & Sin datos \\
\hline \multirow[t]{13}{*}{ Poaceae } & $\begin{array}{c}\text { Cortaderia } \\
\text { speciosa (Nees \& } \\
\text { Meyen) Stapf. }\end{array}$ & Cortadera & $\begin{array}{l}\text { Vega/3300- } \\
3400 \mathrm{~m} \mathrm{s.} \mathrm{m.}\end{array}$ & $\begin{array}{l}\text { Rodríguez } \\
5(\mathrm{SI})\end{array}$ & $\begin{array}{c}\text { No mencionan } \\
\text { usos }\end{array}$ \\
\hline & $\begin{array}{l}\text { Deyeuxia cfr. } \\
\text { curvula Wedd. }\end{array}$ & $\begin{array}{l}\text { Pasto } \\
\text { vicuña }\end{array}$ & $\begin{array}{l}\text { Vega/3300- } \\
3400 \mathrm{~m} \mathrm{s.} \mathrm{m.}\end{array}$ & $\begin{array}{l}\text { Rodríguez } \\
26 \text { (SI) }\end{array}$ & Sin datos \\
\hline & D. chrysophylla Phil. & Sin datos & $\begin{array}{l}\text { Vega/3300- } \\
3400 \mathrm{~m} \mathrm{s.} \mathrm{m.}\end{array}$ & $\begin{array}{l}\text { SI } 28.338, \\
\text { Rodríguez } \\
28(\mathrm{SI})\end{array}$ & Sin datos \\
\hline & D. deserticola Phil. & Sin datos & $\begin{array}{l}\text { Vega/3300- } \\
3400 \mathrm{~m} \mathrm{s.} \mathrm{m.}\end{array}$ & SI s.n. & Sin datos \\
\hline & $\begin{array}{l}\text { D. eminens var. } \\
\text { eminens J. Presl. }\end{array}$ & $\begin{array}{l}\text { Pasto } \\
\text { huailla, } \\
\text { Huaya, Iru, } \\
\text { Sora-Sora }\end{array}$ & $\begin{array}{l}\text { No fue colectada } \\
\text { en el área }\end{array}$ & $\begin{array}{c}\text { Cabrera } \\
8944 \text { (BAA), } \\
\text { Vervoorst } \\
717 \text { (LIL) }\end{array}$ & Forraje \\
\hline & $\begin{array}{l}\text { D. eminens var. fulva } \\
\text { (Griseb.) Rúgolo }\end{array}$ & $\begin{array}{l}\text { Pasto } \\
\text { de vega, } \\
\text { Cebadilla } \\
\text { de vicuña }\end{array}$ & $\begin{array}{l}\text { Vega/3300- } \\
3400 \mathrm{~m} \mathrm{s.} \mathrm{m.}\end{array}$ & $\begin{array}{l}\text { Rodríguez } \\
\text { s.n. (SI) }\end{array}$ & Forraje \\
\hline & $\begin{array}{l}\text { D. rigescens (J. } \\
\text { Presl.) Türpe }\end{array}$ & Sin datos & $\begin{array}{l}\text { Vega/3300- } \\
3400 \mathrm{~m} \mathrm{s.} \mathrm{m.}\end{array}$ & $\begin{array}{l}\text { Rodríguez } \\
27(\mathrm{SI})\end{array}$ & Sin datos \\
\hline & $\begin{array}{l}\text { D. velutina } \\
\text { var. nardifolia } \\
\text { (Griseb.) Phil. }\end{array}$ & Sin datos & $\begin{array}{l}\text { Vega/3300- } \\
3400 \mathrm{~m} \mathrm{s.} \mathrm{m.}\end{array}$ & SI 28.339 & Sin datos \\
\hline & Distichlis sp. & Sin datos & $\begin{array}{l}\text { Vega/3300- } \\
3400 \mathrm{~m} \mathrm{s.} \mathrm{m.}\end{array}$ & SI 28.210 & Forraje \\
\hline & $\begin{array}{c}\text { Festuca } \\
\text { chrysophylla Phil. }\end{array}$ & $\begin{array}{c}\text { Paja } \\
\text { vizcachera }\end{array}$ & $\begin{array}{l}\text { Pajonal sobre meseta/ } \\
>3900-4700 \mathrm{~m} \mathrm{~s} . \mathrm{m} \text {. }\end{array}$ & $\begin{array}{l}\text { Rodríguez } \\
\text { s/n (SI) }\end{array}$ & Forraje \\
\hline & $\begin{array}{c}\text { Festuca } \\
\text { orthophylla Pilg. }\end{array}$ & $\begin{array}{l}\text { Iru, Paja } \\
\text { vizcachera }\end{array}$ & $\begin{array}{l}\text { Pajonal sobre meseta/ } \\
>3900-4700 \mathrm{~m} \mathrm{~s} . \mathrm{m} \text {. }\end{array}$ & $\begin{array}{l}\text { Rodríguez } \\
\text { s/n (SI) }\end{array}$ & Forraje \\
\hline & $\begin{array}{l}\text { Festuca villipalea } \\
\text { (St.-Yves) E. } \\
\text { B. Alexeev }\end{array}$ & Sin datos & $\begin{array}{l}\text { Pajonal sobre meseta/ } \\
>3900-4700 \mathrm{~m} \mathrm{~s} . \mathrm{m} \text {. }\end{array}$ & $\begin{array}{l}\text { Rodríguez } \\
7 \text { y } 8 \text { (SI) }\end{array}$ & Forraje, techos \\
\hline & F. weberbaueri Pilg. & $\begin{array}{c}\text { Paja } \\
\text { vizcachera } \\
\text { (Haber, } \\
\text { 1987) }\end{array}$ & $\begin{array}{l}\text { Pajonal sobre meseta/ } \\
>3900-4700 \mathrm{~m} \mathrm{~s} . \mathrm{m} \text {. }\end{array}$ & $\begin{array}{l}\text { Rodríguez } 6 \\
7 \text { y } 9(\mathrm{SI})\end{array}$ & Forraje, techos \\
\hline
\end{tabular}


Bol. Soc. Argent. Bot. 54 (1) 2019

\begin{tabular}{|c|c|c|c|c|c|}
\hline Familia & Nombre científico & $\begin{array}{c}\text { Nombre } \\
\text { vernáculo }\end{array}$ & $\begin{array}{c}\text { Asociación } \\
\text { vegetación / altitud }\end{array}$ & $\begin{array}{l}\text { Ejemplar de } \\
\text { referencia }\end{array}$ & Uso actual \\
\hline \multirow[t]{4}{*}{ Poaceae } & $\begin{array}{l}\text { Pappostipa frigida } \\
\text { (Phil) Romasch }\end{array}$ & Sin datos & $\begin{array}{l}\text { Pajonal/ >3900 } \\
-4700 \text { m s. m. }\end{array}$ & $\begin{array}{l}\text { SI 28.340, } \\
\text { SI 28.341, } \\
\text { SI } 28.342\end{array}$ & Forraje, techos \\
\hline & $\begin{array}{l}\text { P. vaginata (Phil.) } \\
\text { Romasch }\end{array}$ & Sin datos & $\begin{array}{l}\text { Pajonal sobre meseta/ } \\
>3900-4700 \text { m s. m. }\end{array}$ & $\begin{array}{l}\text { Rodríguez } \\
25(\mathrm{SI})\end{array}$ & Forraje, techos \\
\hline & $\begin{array}{c}\text { Polypogon } \\
\text { interruptus Kunth. }\end{array}$ & Sin datos & $\begin{array}{l}\text { Pajonal/ >3900 } \\
-4700 \text { m s. m. }\end{array}$ & SI 28.33 & Forraje \\
\hline & $\begin{array}{l}\text { Puccinellia frigida } \\
\text { (Phil) I. M. Johnst. }\end{array}$ & & $\begin{array}{l}\text { Pajonal/ >3900 } \\
-4700 \text { m s. m. }\end{array}$ & & \\
\hline Portulacaceae & $\begin{array}{l}\text { Cistanthe minuscula } \\
\text { (Añon) Peralta }\end{array}$ & Sin datos & Tolar/3400-3900 m s. m. & SI 28.207 & Sin datos \\
\hline \multirow[t]{6}{*}{ Solanaceae } & $\begin{array}{c}\text { Fabiana } \\
\text { bryoides Phil. }\end{array}$ & $\begin{array}{l}\text { Pata de } \\
\text { perdiz }\end{array}$ & $\begin{array}{c}\text { Tolar/3400-3900 m } \\
\text { s. m. y laderas de la } \\
\text { quebrada/ } 4000 \text { m s.m. }\end{array}$ & SI 28.287 & $\begin{array}{l}\text { Combustible, } \\
\text { infusiones, } \\
\text { sahumador, } \\
\text { delimita corrales }\end{array}$ \\
\hline & F. denudata Miers. & Tolilla & Tolar/3400-3900 m s. m. & SI 28.328 & Sin datos \\
\hline & $\begin{array}{l}\text { F. punensis S. } \\
\text { C. Arroyo }\end{array}$ & Tolilla & Tolar/3400-3900 m s. m. & $\begin{array}{l}\text { Rodríguez } \\
17(\mathrm{SI})\end{array}$ & $\begin{array}{l}\text { Combustible, } \\
\text { forrajera, } \\
\text { medicinal }\end{array}$ \\
\hline & Lycium chanar Phil. & Acerillo & Tolar/3400-3900 m s. m. & $\begin{array}{l}\text { Rodríguez } \\
14(\mathrm{SI})\end{array}$ & Sin datos \\
\hline & $\begin{array}{l}\text { Nicotiana petunioides } \\
\text { (Griseb.) MIllán }\end{array}$ & $\begin{array}{l}\text { Tabaco } \\
\text { de burro }\end{array}$ & Tolar/3400-3900 m s. m. & $\begin{array}{l}\text { Rodríguez } \\
4(\mathrm{SI})\end{array}$ & $\begin{array}{c}\text { Forrajera } \\
\text { (Haber, 1987) }\end{array}$ \\
\hline & $\begin{array}{l}\text { N. undulata } \\
\text { Ruíz \& Pav. }\end{array}$ & Sin datos & Tolar/3400-3900 m s. m. & SI 28.215 & Sin datos \\
\hline \multirow[t]{3}{*}{ Verbenaceae } & $\begin{array}{c}\text { Acantholippia } \\
\text { deserticola (Phil.) } \\
\text { Moldenke }\end{array}$ & Rica-rica & Tolar/3400-3900 m s. m. & $\begin{array}{l}\text { Rodríguez } \\
1(\mathrm{SI})\end{array}$ & $\begin{array}{l}\text { Medicinal, } \\
\text { combustible }\end{array}$ \\
\hline & A. salsoloides Griseb. & Rica-rica & Tolar/3400-3900 m s. m. & SI 28.218 & $\begin{array}{l}\text { Medicinal, } \\
\text { combustible }\end{array}$ \\
\hline & $\begin{array}{c}\text { Neosparton } \\
\text { ephedroides Griseb. }\end{array}$ & Badre & Tolar/3400-3900 m s. m. & $\begin{array}{l}\text { Rodríguez } \\
12(\mathrm{SI})\end{array}$ & Sin datos \\
\hline
\end{tabular}

media y cañas floríferas con órganos reproductivos o sin ellos. Se colorearon con doble coloración (safranina-verde rápido) (D'Ambrogio de Argüeso, 1986; Zarlavsky, 2014). Por último, estos materiales fueron observados y fotografiaron con MO Nikon Eclipse E200.

En todos los casos, la identificación de los macrorrestos (leñosos, carbonizados o no, y herbáceos) se realizó por comparación morfológica $\mathrm{y}$ anatómica a partir del material de referencia coleccionado en el área de estudio, determinado y depositado en el Herbario del Instituto de Botánica Darwinion (SI) y, en algunos casos, se utilizaron especímenes de dicho Herbario. Al tratarse de material ya identificado no fue necesario el uso de claves taxonómicas. Para el estudio morfológico se utilizaron las plantas completas depositadas en SI, mientras que para el análisis anatómico se recurrió a la histoteca de referencia mencionada más arriba.

\section{Resultados}

El material arqueobotánico recuperado y analizado consiste en macrorrestos herbáceos (pétalos, flores, estambres con polen preservado, semillas, frutos, hojas, tallos herbáceos, inflorescencias, espinas), leñosos no carbonizados (tallos y raíces leñosos) y leñosos carbonizados (carbones) (Tabla 3; Fig. 2A-D).

Las familias Asteraceae, Brassicaceae, Cactaceae, Caryophyllaceae, Chenopodiaceae, 


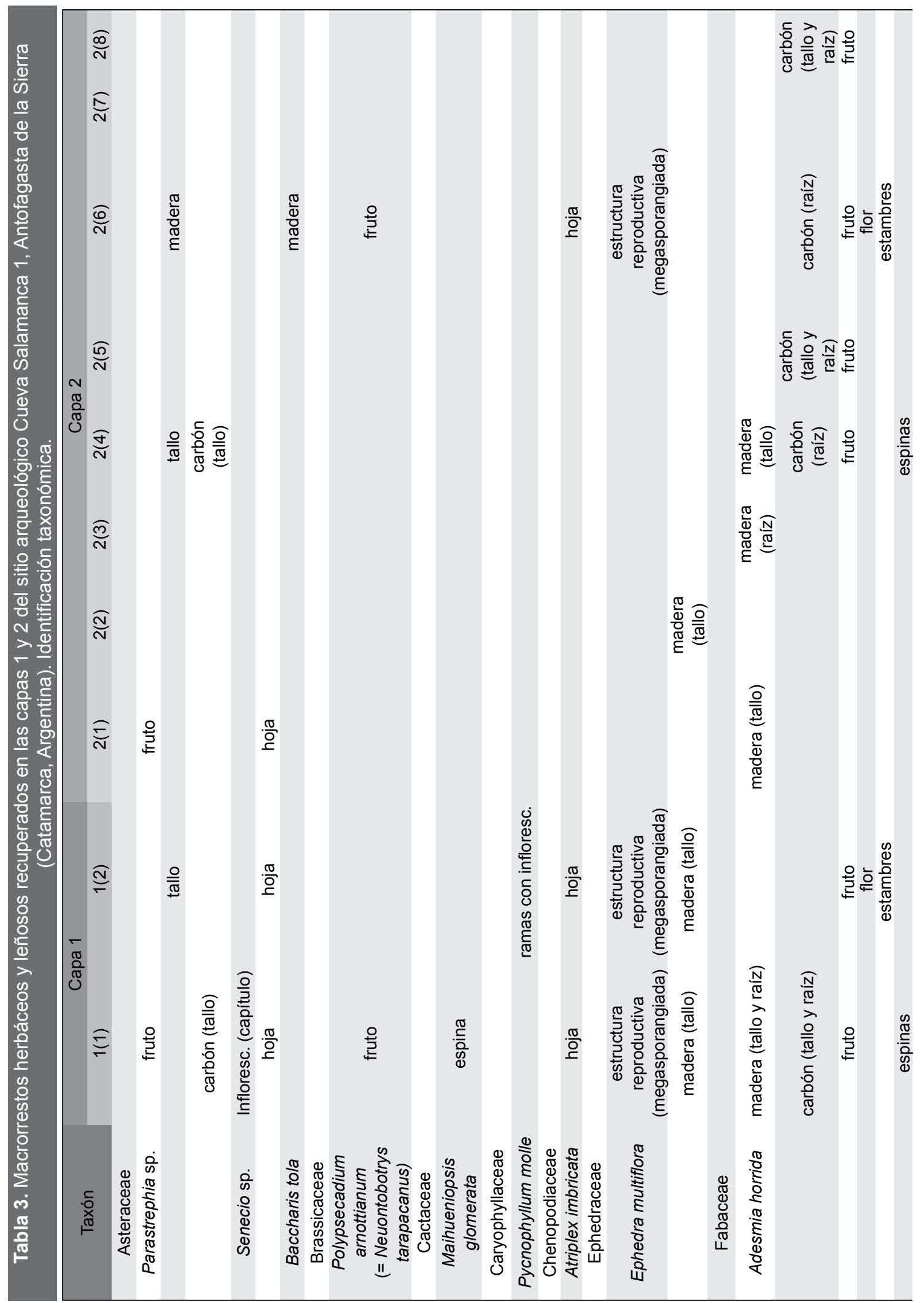


Bol. Soc. Argent. Bot. 54 (1) 2019

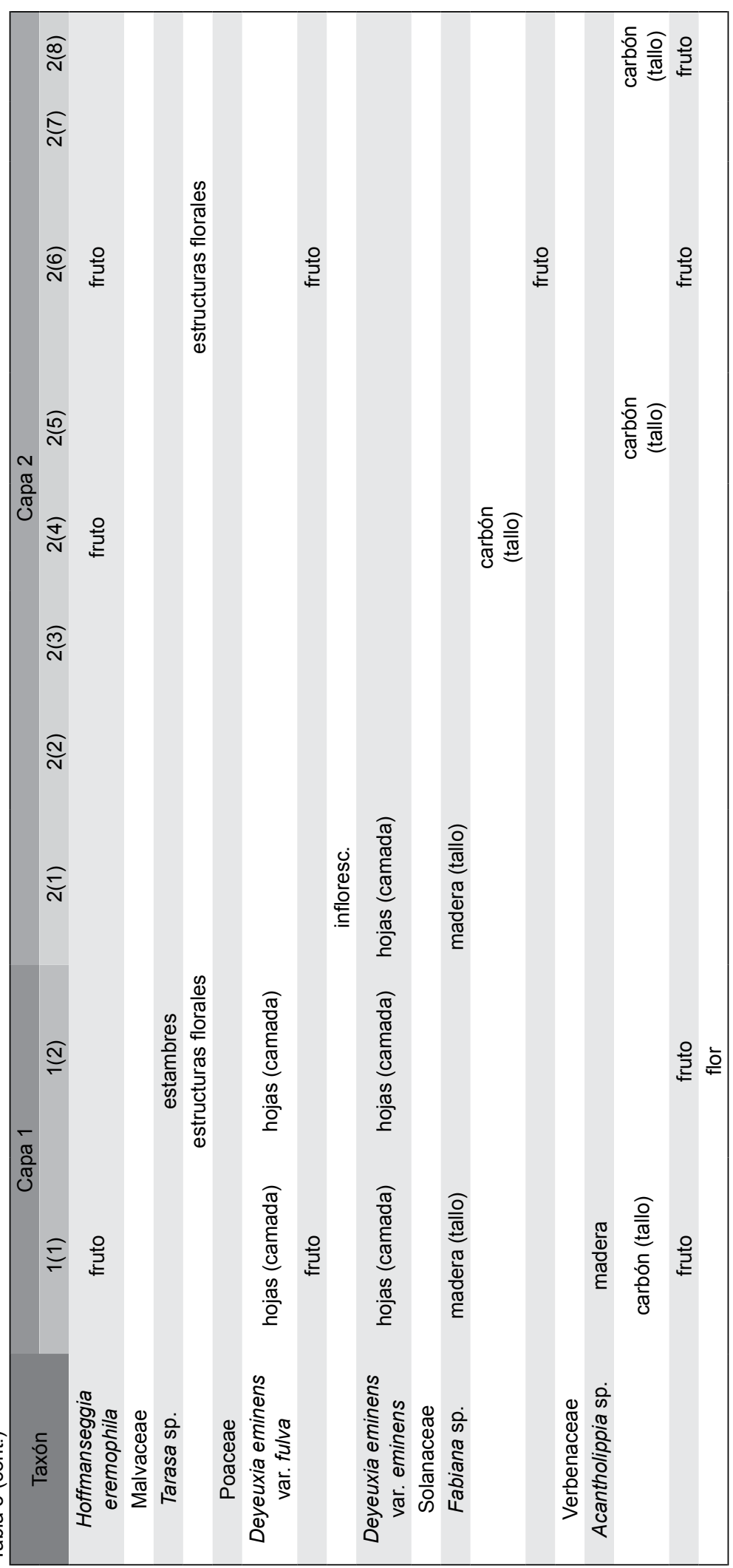




\section{N. M. Apóstolo et al. - Macrorrestos vegetales en el sitio arqueológico Cueva Salamanca 1}
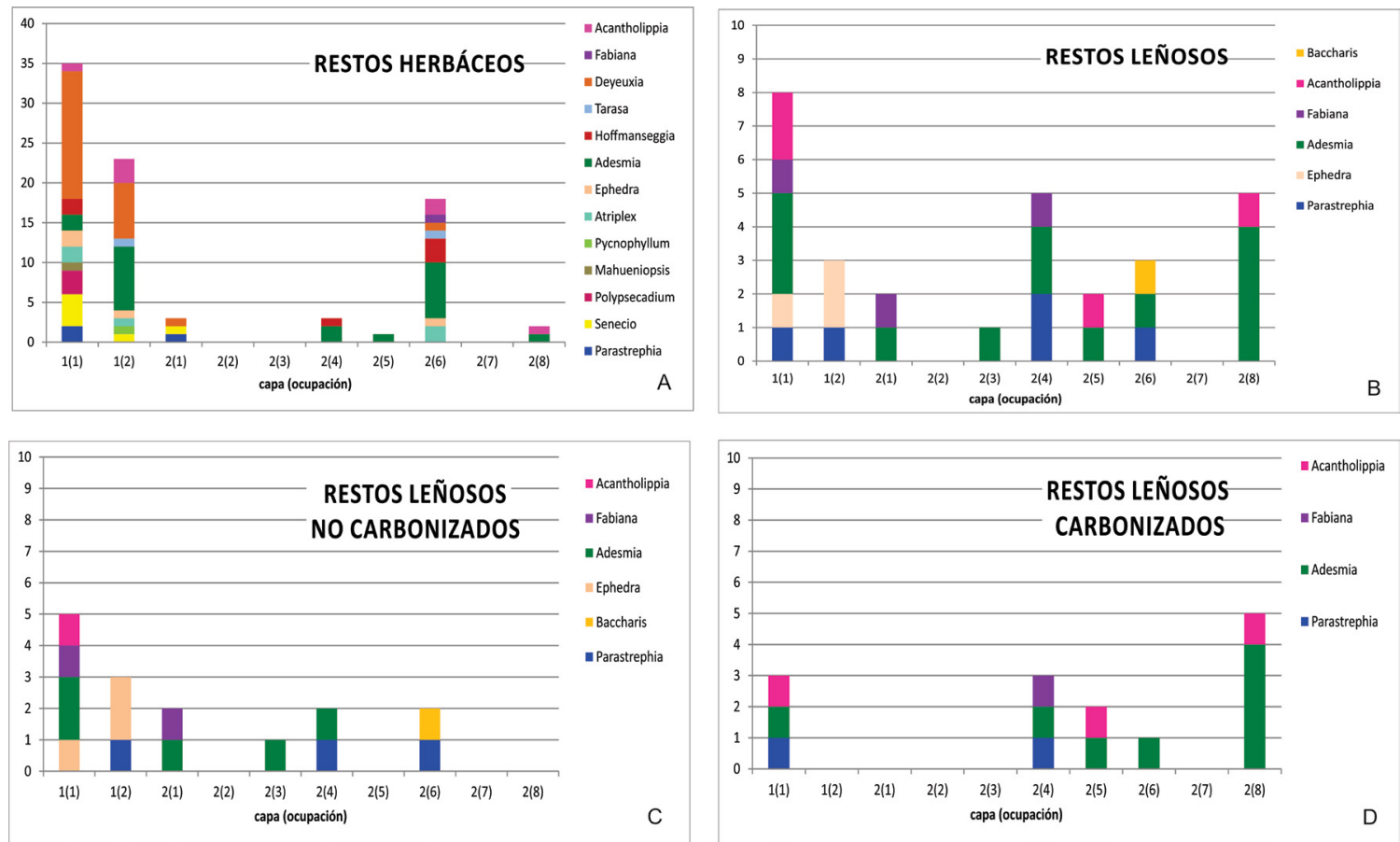

Fig. 2. Frecuencia de especies identificadas a partir del muestreo de los macrorrestos vegetales recuperados en el sitio Cueva Salamanca 1, Antofagasta de la Sierra (Catamarca, Argentina). A: Macrorrestos herbáceos totales (frutos, semillas, restos florales, hojas y tallos herbáceos). B: Macrorrestos leñosos totales. C: Macrorrestos leñosos no carbonizados (tallos y raíces leñosas). D: Macrorrestos leñosos carbonizados (carbones).

Ephedraceae, Fabaceae, Malvaceae, Poaceae, Solanaceae y Verbenaceae conforman el registro arqueobotánico de CS1 (Tabla 3). La mayor cantidad de macrorrestos, tanto herbáceos como leñosos, fueron hallados en las capas 1(1) y 1(2) de la Estructura 3. También se observa una importante diversidad de restos leñosos en 2(4) y 2(6) (Tabla 3; Fig. 2B-D). Las ocupaciones de la capa 2 exhiben baja diversidad de restos herbáceos, excepto en 2(6) (Tabla 3; Fig. 2A). Independientemente de su presencia en cada nivel, los restos herbáceos de los géneros Adesmia sp. (Fabaceae) y Deyeuxia sp. (Poaceae) son los más abundantes (Tabla 3; Fig. 2A-D). El primer género está representado por restos florales (pétalos, estilos, estambres con polen preservado), porciones de sus frutos (artejos) y restos de tallos (Tabla 3; Fig. 3B-F). El segundo es muy abundante y conforma las camadas de gramíneas que constituyen los pisos de ocupación en las capas 1(1) y 1(2), (Tabla 3; Fig. 4B-F). Dichas camadas están compuestas por hojas de
Deyeuxia eminens var. fulva (vainas y láminas) (Fig. 4D), hojas de Deyeuxia eminens var. eminens (Fig. 4E-F) y frutos de este género (Fig. 4B).

Asimismo, se identificaron otros tipos de restos herbáceos con menor representación (Tabla 3; Fig. 2A). En este sentido, se registró la presencia de restos de inflorescencia (capítulo) y de hojas de Senecio sp. (Asteraceae) en las capas 1(1), 1(2) y 2(1) (Tabla 3; Fig. 5A, B), porciones de frutos de Brassicaceae, probablemente pertenecientes a Polypsecadium arnottianum o Neuontobotrys tarapacanus en las capas 1(1) y 2(6) (Tabla 3, Fig. 5D) y frutos completos de Hoffmanseggia eremophila (Fabaceae) en las capas 1(1), 2(4) y 2(6) (Tabla 3; Fig. 3A). Los restos florales y frutos completos con semillas correspondientes al género Acantholippia (Verbenaceae) fueron recuperados en las capas 1(2), 2(6) y 2(8) (Tabla 3; Fig. 6F-G). Es de destacar que todos los frutos de este género fueron hallados cerrados $\mathrm{y}$, al ser abiertos experimentalmente, liberaron su semilla 
Bol. Soc. Argent. Bot. 54 (1) 2019
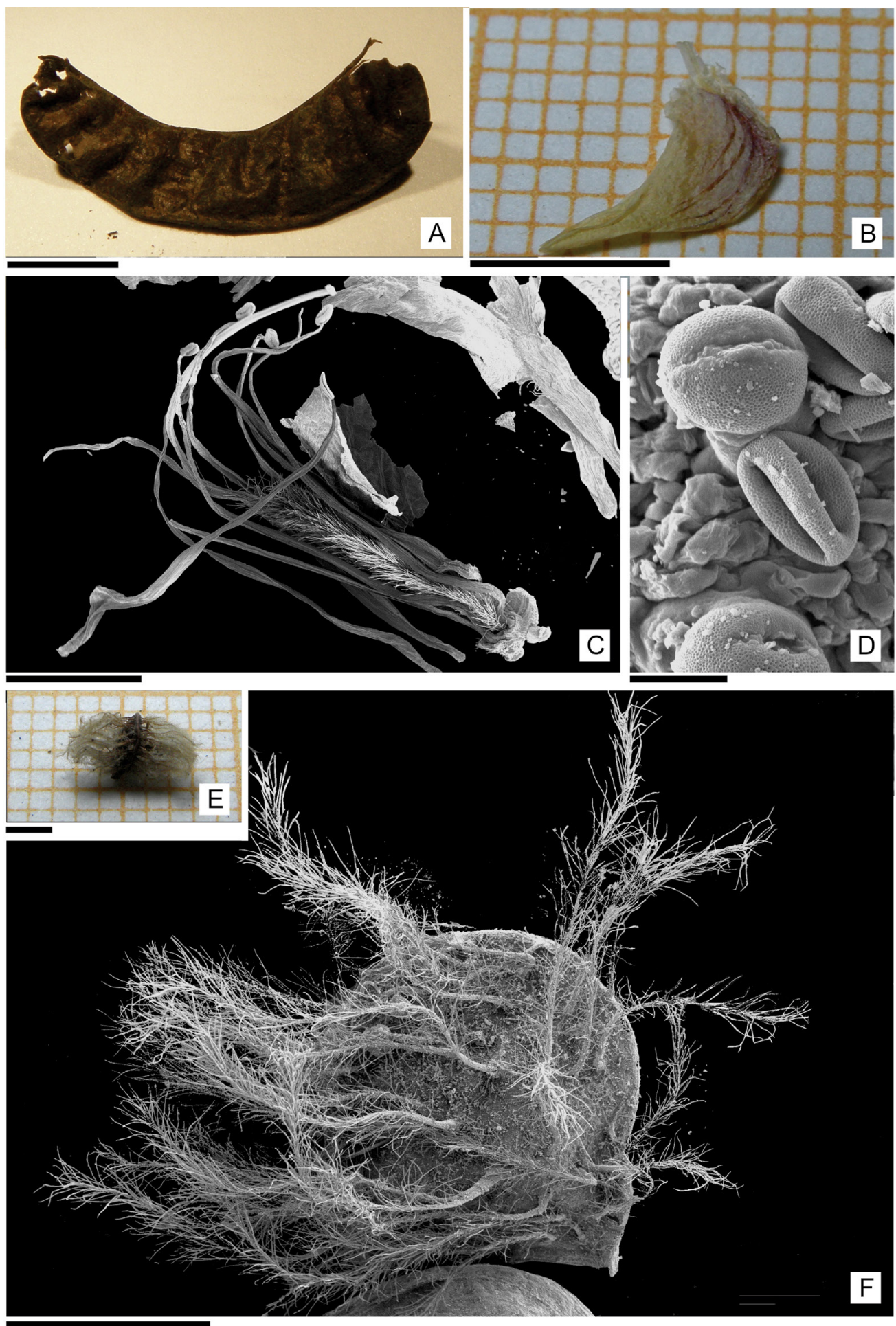

Fig. 3. Macrorrestos herbáceos recuperados en el sitio arqueológico Cueva Salamanca 1, Antofagasta de la Sierra (Catamarca, Argentina). A: Hoffmanseggia eremophila (Fabaceae), fruto. B-D: Adesmia sp. (Fabaceae). B: Pétalo. C: Flor; D. Estambre con polen preservado. E - F: Artejo de fruto. Escalas= A, B: 5 $\mathrm{mm} ; \mathrm{C}, \mathrm{E}, \mathrm{F}: 2 \mathrm{~mm}$; D: $10 \mu \mathrm{m}$. 

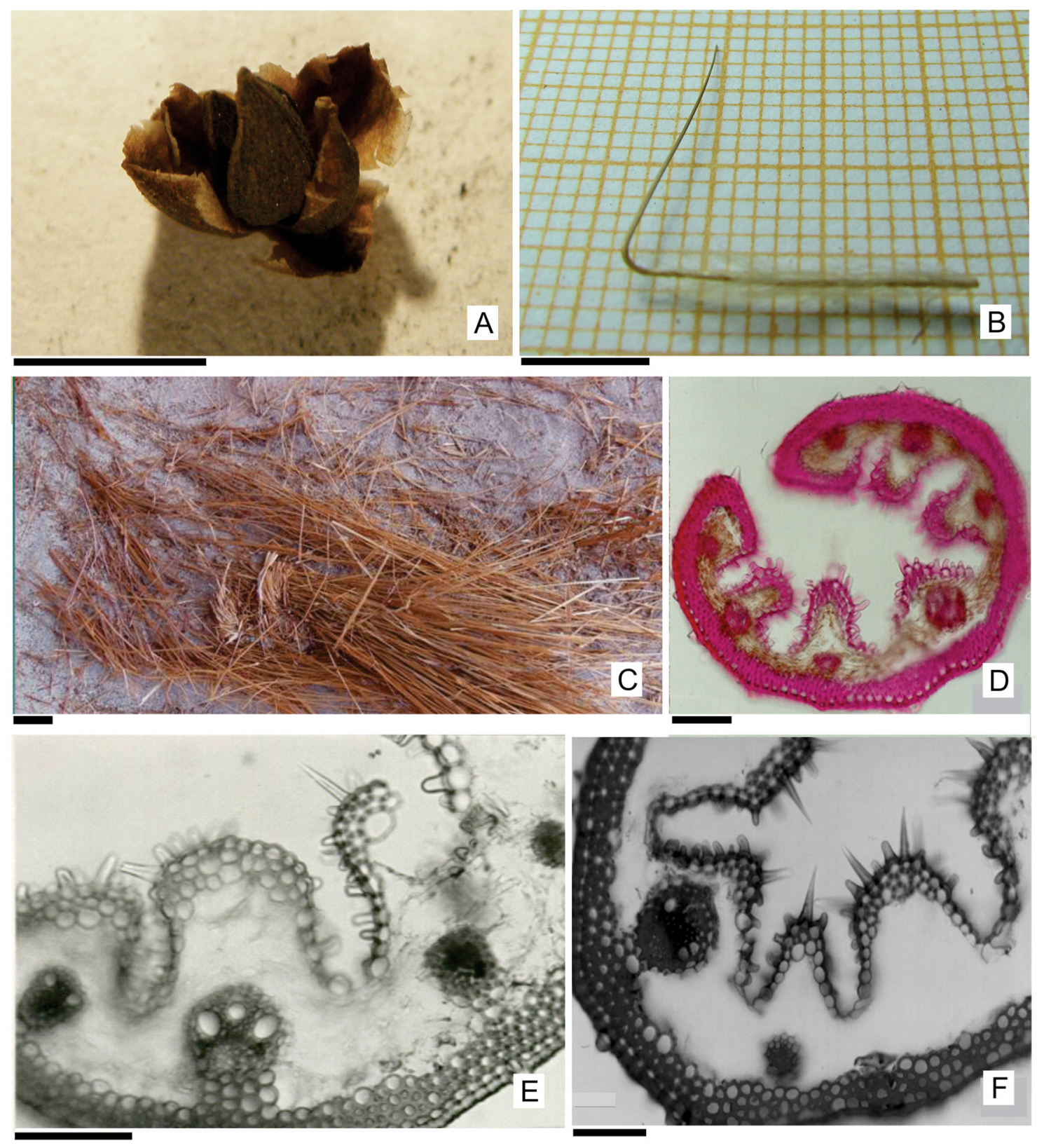

Fig. 4. Macrorrestos herbáceos recuperados en el sitio arqueológico Cueva Salamanca 1, Antofagasta de la Sierra (Catamarca, Argentina). A: Ephedra sp. (Ephedraceae), estructura reproductiva. B-F: Deyeuxia eminens (Poaceae). B: Fruto. C: Camadas de hojas. D: Corte transversal de hoja de D. eminens var. fulva, material arqueológico. E-F: Corte transversal de hoja de $D$. eminens var. eminens. E: Material actual. F: Material arqueológico. Escalas =A, B: $5 \mathrm{~mm}$; C: $5 \mathrm{~cm}$; D-F: $100 \mu \mathrm{m}$.

y emitieron un agradable aroma, típico de la mencionada familia.

Finalmente, fueron determinados algunos macrorrestos herbáceos con baja representación en las capas 1(1), 1(2) y 2(6) (Tabla 3; Fig. 2A), como Ephedra (Fig4A), Parastrephia (Fig. 5C), Atriplex(Fig. 5E, F), Tarasa (Fig. 6B-E) y Pycnophyllum (Fig. 6A), así como también una 
Bol. Soc. Argent. Bot. 54 (1) 2019
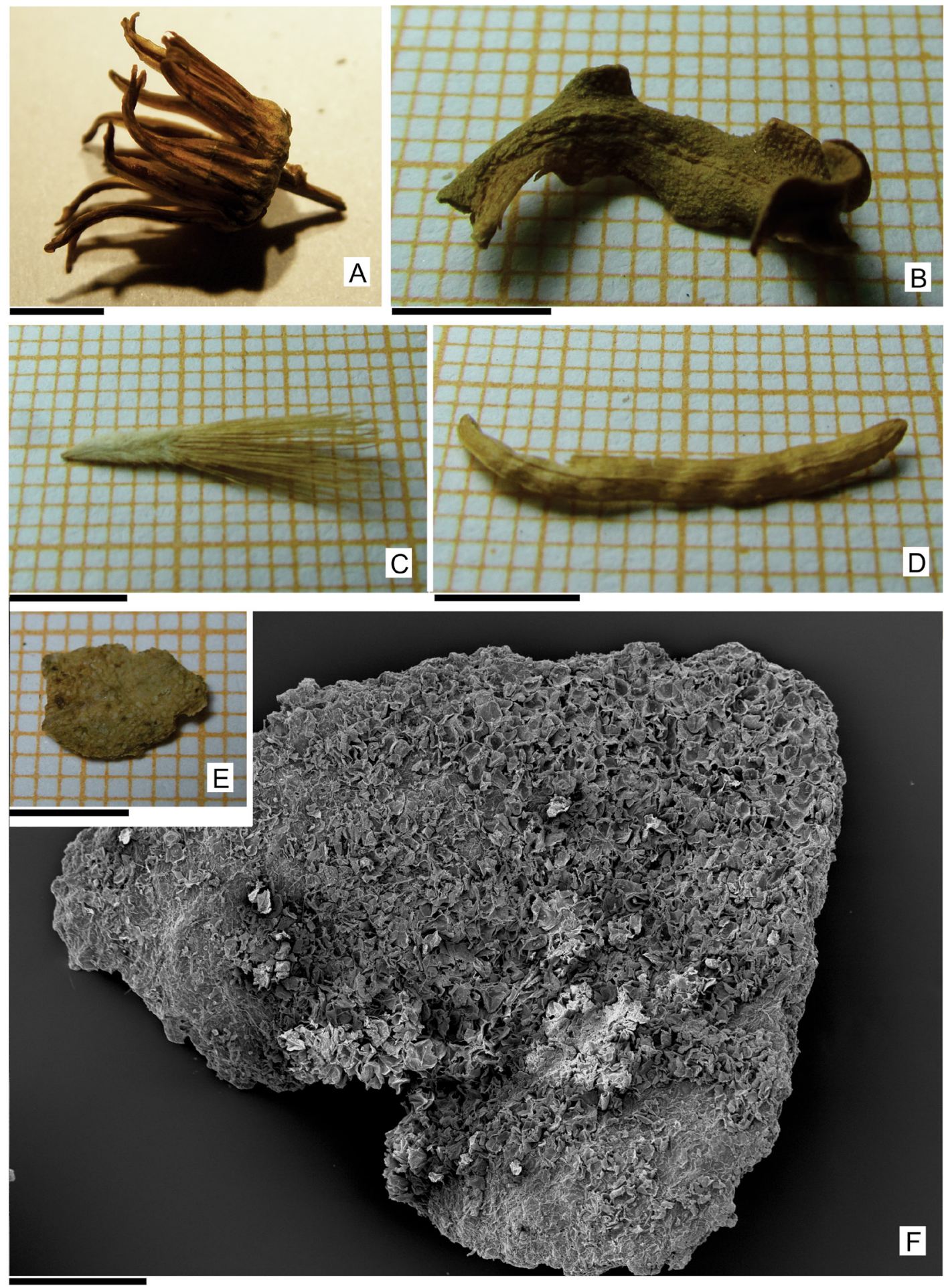

Fig. 5. Macrorrestos herbáceos recuperados en el sitio arqueológico Cueva Salamanca 1, Antofagasta de la Sierra (Catamarca, Argentina). A-B: Senecio sp. (Asteraceae). A: Inflorescencia (capítulo). B: Hoja. C: Parastrephia sp. (Asteraceae), fruto (aquenio). D: Brassicaceae, porción de fruto. E-F: Atriplex imbricata (Chenopodiaceae), hoja. Escalas = A-F: $5 \mathrm{~mm}$. 

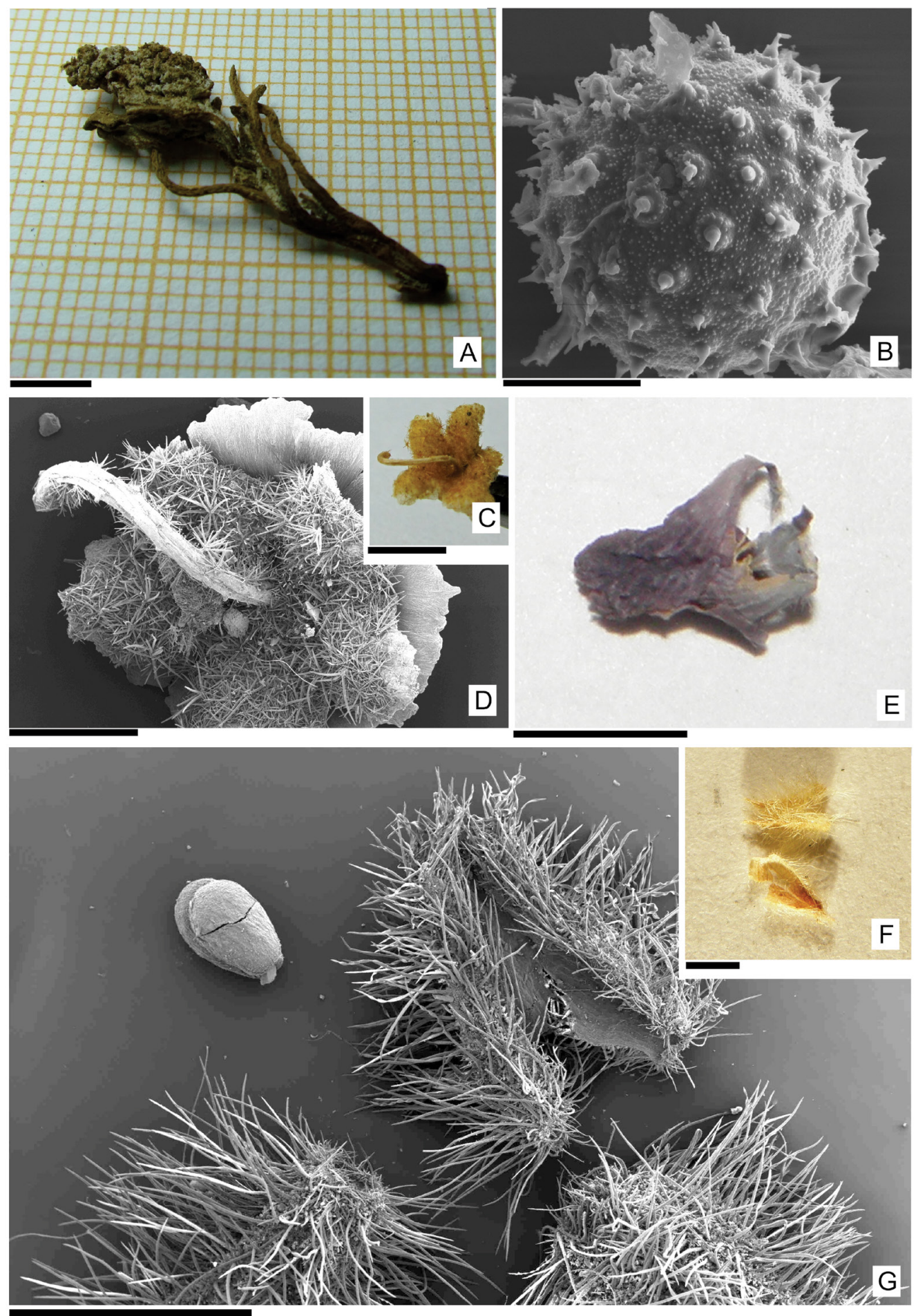

Fig. 6. Macrorrestos herbáceos recuperados em elsitio arqueológico Cueva Salamanca 1, Antofagasta de la Sierra (Catamarca, Argentina). A. Pycnophyllum molle (Caryophyllaceae), rama e inflorescencia. B-D: Tarasa sp. (Malvaceae). B: Polen recuperado de un estambre. C-D: Restos florales. E: Pétalos. F-G: Acantholippia sp. (Verbenaceae), frutos y semillas. Escalas = A, C, E: 5 mm; B: $20 \mu \mathrm{m}$; D, F, G: 2 mm. 
espina de Maihueniopsis glomerata. Por otra parte, los restos leñosos recuperados exhiben una diversidad algo menor en cuanto a cantidad de representantes. Se registró la presencia de las familias Verbenaceae, Solanaceae, Fabaceae, Ephedraceae y Asteraceae (Tabla 3; Fig. 2B-D).

La mayor diversidad de restos leñosos se observa en las capas 2(1) y 2(2) siendo proporcional la cantidad entre restos carbonizados y no carbonizados. Las restantes ocupaciones exhiben entre una a tres especies (Fig. 2B-D). El género Ephedra (Ephedraceae) sólo está representado por restos de madera no carbonizada en las capas 1(1), 1(2) y 2(2) (Tabla 3; Figs. 7H, 8A). Baccharis tola (Asteraceae) fue identificada en una porción de madera no carbonizada de tallo localizada en la capa 2(6) (Tabla 3; Figs. 7E, 9A-B), mientras que los géneros Acantholippia (Verbenaceae), Parastrephia (Asteraceae), Adesmia (Fabaceae) y Fabiana (Solanaceae) presentan restos carbonizados y no carbonizados (Figs. 2B-D; 7AD, F-G; 8B-F; 9C-F). Es de destacar que Adesmia sp. se identificó en casi todas las ocupaciones de las capas 1 y 2 y, es el taxón que más representación tiene en los materiales carbonizados (Figs. 2B-D; $9 \mathrm{E}-\mathrm{F})$. Incluso en la ocupación más temprana con registro de especies vegetales -2(8)- es el único material leñoso carbonizado (Tabla 3; Fig. 2D). En líneas generales, las capas 2(4) a 2(8) presentan una importante cantidad de macrorrestos carbonizados, mientras que los macrorrestos no carbonizados se hallaron principalmente en las ocupaciones más tardías (Tabla 3; Fig. 2B-D).

\section{Discusión y Conclusiones}

A partir de los resultados obtenidos, es posible afirmar que, de las aproximadamente 60 especies vegetales que crecen en el ambiente actual (Cuello, 2006; Rodríguez, 2013) relacionado al sitio arqueológico CS1, se recuperó el $44 \%$ en las capas 1 y 2 del sitio. Dado que los representantes de las distintas familias hallados en el sitio CS1 crecen en el tolar, el pajonal y la vega, puede considerarse que la paleovegetación era similar a la actual en el área de estudio (Tablas 2 y 3 ). En cuanto a la proporción de taxones recuperados, es posible que se trate de aquellos que fueron más útiles en CS1 como base residencial (combustible, camadas en áreas de descanso, entre otros) y de aquellos que se incorporaron por causas naturales, tales como los restos florales.

Es importante hacer referencia a las adaptaciones morfológicas y anatómicas observadas en las especies halladas que reflejan las condiciones del paleoambiente durante el Holoceno. Las capas 2(10) a 2(3) corresponden al Holoceno Medio temprano (sensu Yacobaccio, 2013), más cálido y seco que el Holoceno Temprano, mientras que las capas 2(2) y 2(1) están asociadas al Holoceno Medio tardío, durante el cual se incrementa la aridez y la fragmentación del ambiente; por último, las capas1(1) y 1(2) corresponden al Holoceno Tardío, período en el que comienzan a establecerse las condiciones climáticas semejantes a las actuales en ANS, $c a$. 3.600 AP (Tabla 1).

Teniendo en cuenta esta secuencia paleoambiental, se observan variaciones en cuanto a la frecuencia de uso de las distintas familias (Tabla 3; Fig. 2). Durante el Holoceno Medio temprano fue muy abundante el género Adesmia (Fabaceae) y, en menor cantidad, Acantholippia (Verbenaceae), ambos utilizados como combustible. Por otra parte, en el registro arqueobotánico del Holoceno Medio tardío -capas 2 (2) y 2 (1)- son frecuentes las familias Asteraceae, Fabaceae y Solanaceae, representados por flores, inflorescencias y estambres con polen preservado que pueden deber su presencia en el sitio CS1 a causas naturales (Tablas 2 y 3). Por último, las capas correspondientes al Holoceno Tardío -1(2) y 1(1)- son las que exhiben mayor cantidad y diversidad de macrorrestos vegetales. En estas ocupaciones están representadas las familias Asteraceae, Brassicaceae, Cactaceae, Caryophyllaceae, Chenopodiaeae, Ephedraceae, Fabacece, Malvaceae, Poaceae, Solanaceae y Verbenaceae (Tabla 3; Fig. 2), es decir, todas las que fueron identificadas en el muestreo realizado en las cuadrículas de CS1 analizadas. No obstante, la capa 2(6), ca. 7.500 AP correspondiente al Holoceno Medio temprano, presenta una importante cantidad de macrorrestos. Esto podría estar indicando un pequeño intervalo de mayor ocupación del sitio, el cual coincidiría con condiciones climáticas de mayor humedad previas a los momentos más áridos del Holoceno Medio tardío.

A partir de estas consideraciones, es interesante destacar que la mayoría de las especies recuperadas en las capas 2(8) a 2(3) -Holoceno Medio temprano- 

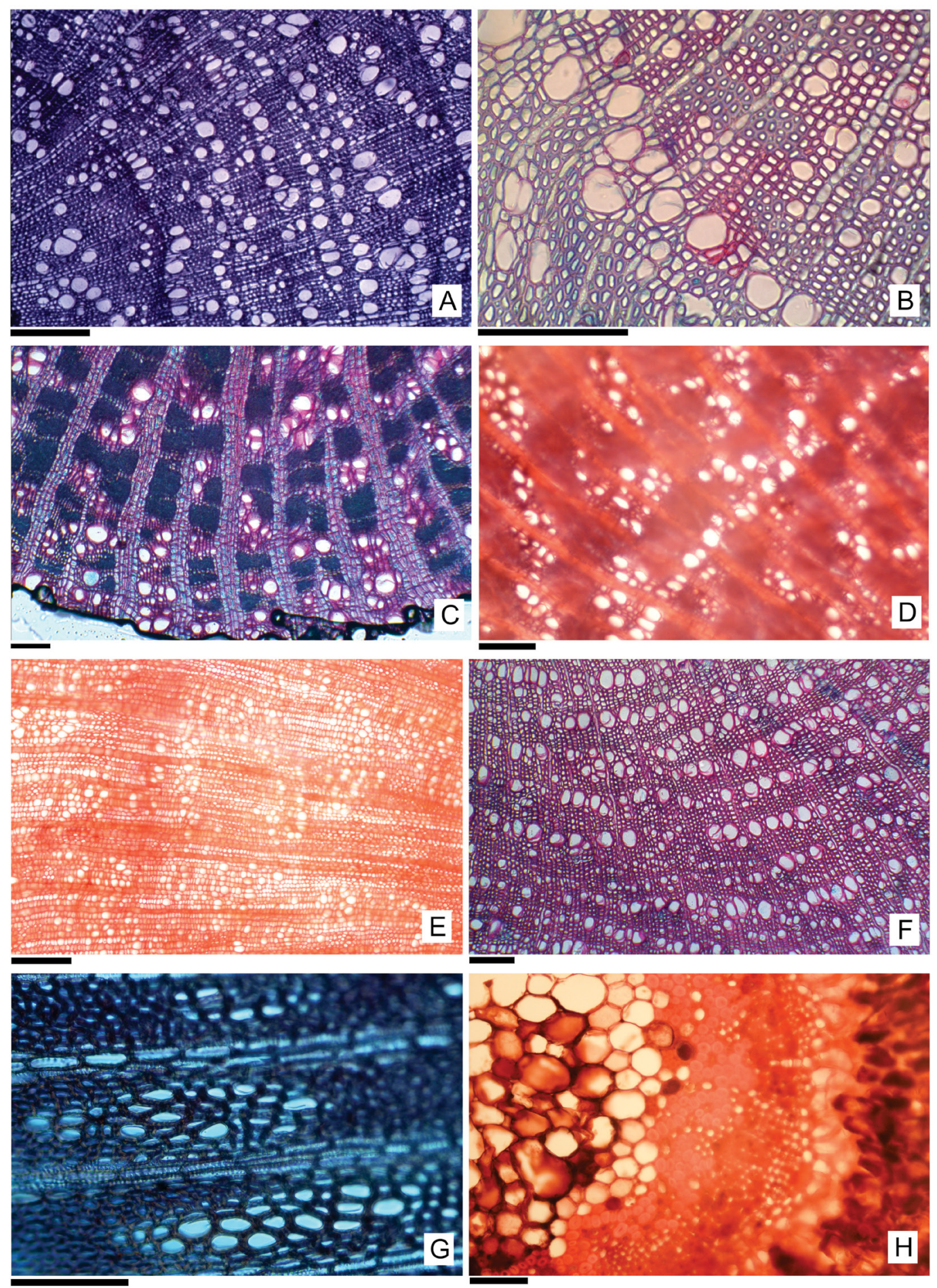

Fig. 7. Materiales de referencia. Maderas actuales, corte transversal. A: Acantholippia deserticola (Verbenaceae). B: A. salsoloides (Verbenaceae). C-D: Adesmia horrida (Fabaceae). C. Raíz. D.Tallo. E. Baccharis tola (Asteraceae). F. Fabiana bryoides (Solanaceae). G. Parastrephia lucida (Asteraceae). H. Ephedra multiflora (Ephedraceae). Escalas = A-G: $100 \mu \mathrm{m}$. 
Bol. Soc. Argent. Bot. 54 (1) 2019
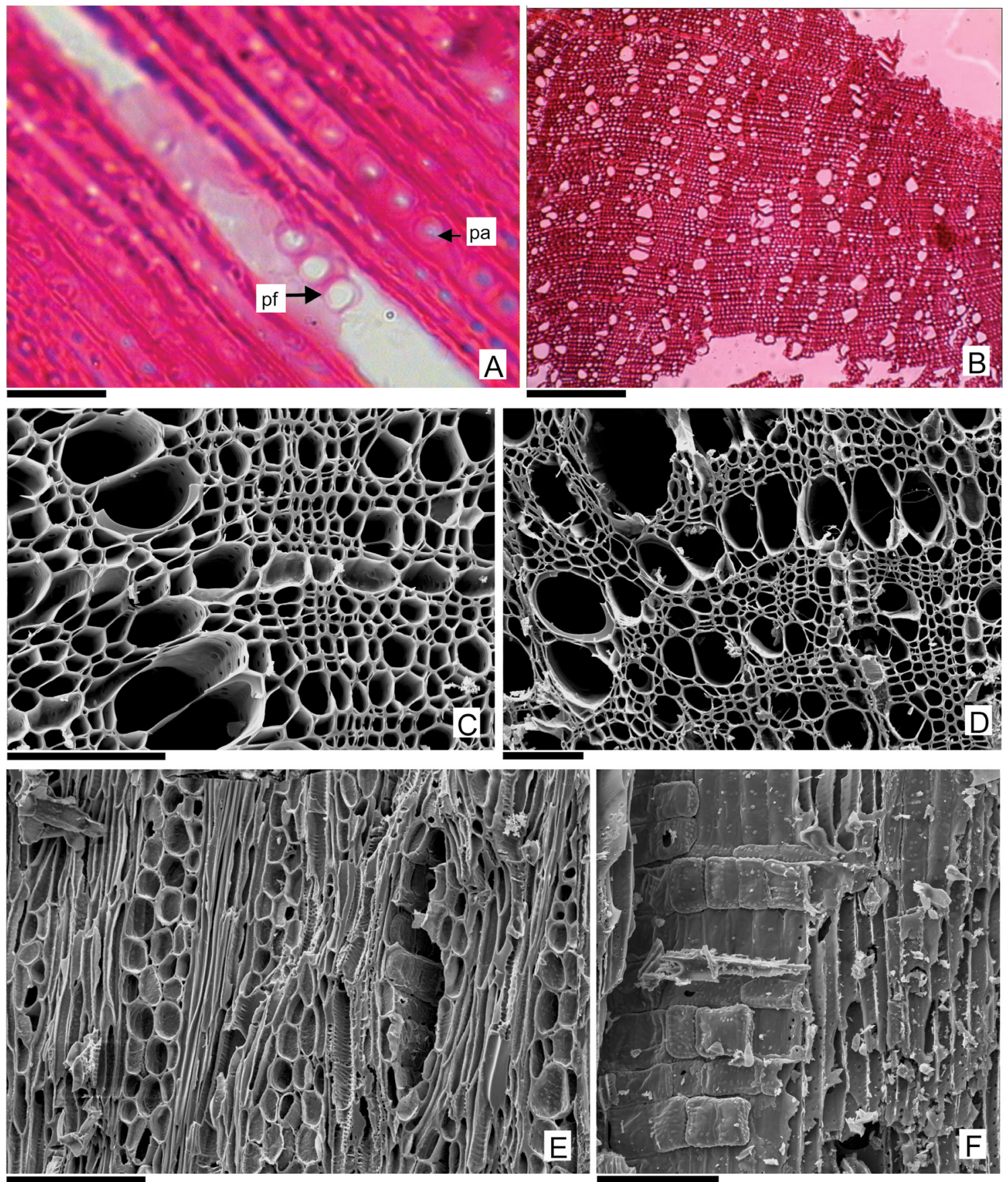

Fig. 8. Macrorrestos leñosos recuperados en el sitio arqueológico Cueva Salamanca 1, Antofagasta de la Sierra (Catamarca, Argentina). A: Ephedra sp. (Ephedraceae), corte longitudinal de tallo. B-C: Fabiana sp. (Solanaceae). B: corte transversal de tallo. C: detalle de corte transversal de carbón. D-F: aff. Acantholippia salsoloides (Verbenaceae), carbón. D: Corte transversal. E: Corte longitudinal tangencial. F: Corte longitudinal radial. Abreviaturas: pa, punteaduras areoladas; pf, placa perforada foraminada. Escalas $=\mathrm{A}$, B, E: $100 \mu \mathrm{m} ; \mathrm{C}, \mathrm{D}, \mathrm{F}: 50 \mu \mathrm{m}$. 

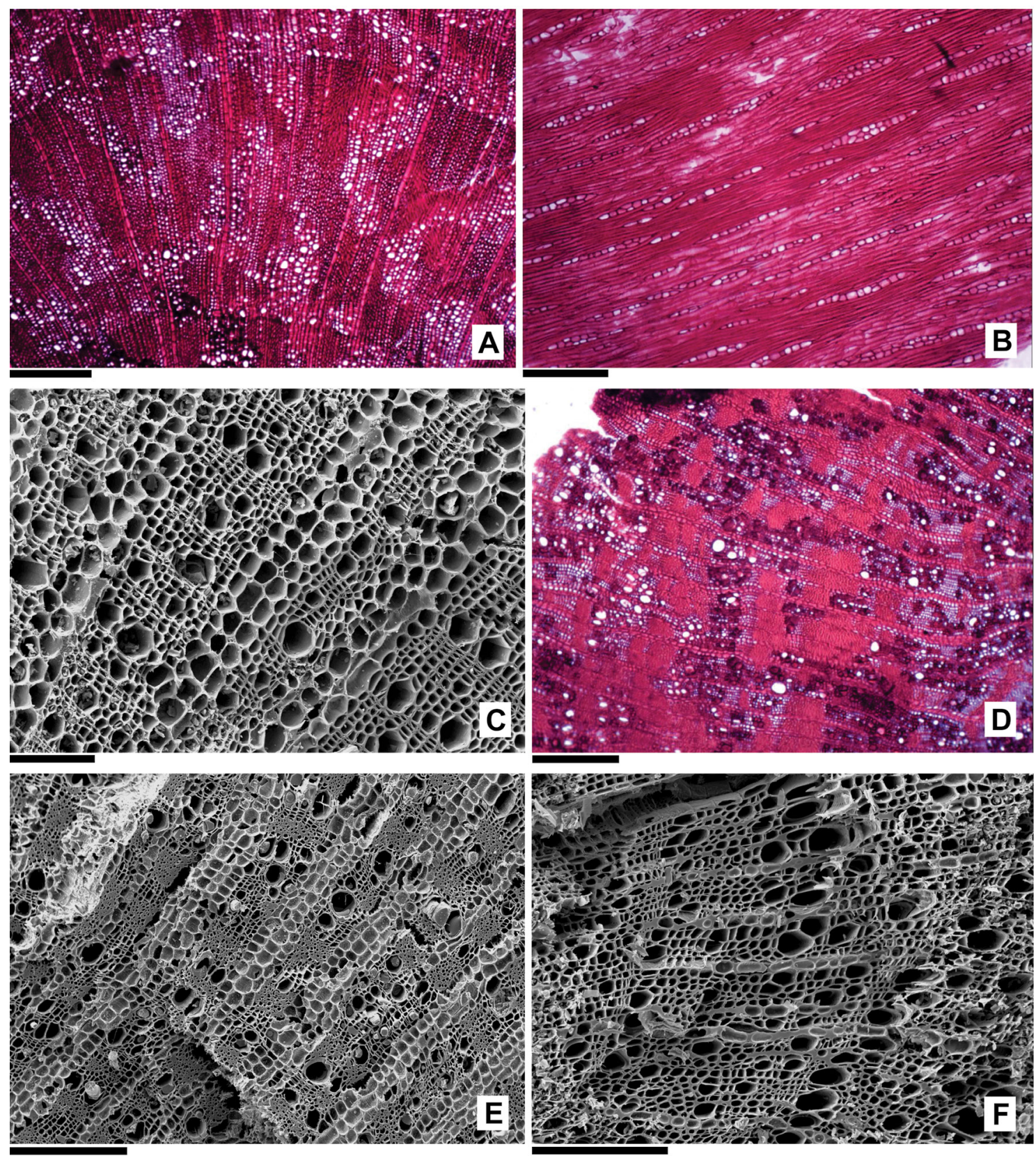

Fig. 9. Macrorrestos leñosos recuperados en el sitio arqueológico Cueva Salamanca 1, Antofagasta de la Sierra (Catamarca, Argentina). A-B: Baccharis tola (Asteraceae), tallo. A: Corte transversal. B: Corte longitudinal tangencial. C: Parastrephia sp. (Asteraceae), corte transversal de tallo (carbón). D-F: aff. Adesmia horrida (Fabaceae). D: Corte transversal de raíz. E: Corte transversal de raíz (carbón). F: Corte transversal de tallo (carbón). Escalas = A-D, F: $100 \mu \mathrm{m} ; \mathrm{E}, 200 \mu \mathrm{m}$. 


\section{Bol. Soc. Argent. Bot. 54 (1) 2019}

presentan caracteres xeromórficos dado que tienen particularidades morfo-anatómicas similares a los observados en las plantas xerófitas pero que pueden adaptarse a ambientes secos y menos áridos tal como ocurre en las plantas acharrapadas o en forma de cojín. Por otra parte, en las ocupaciones correspondientes al Holoceno Medio tardío son frecuentes las especies xerófitas con características que responden a condiciones de gran aridez, donde el agua es el factor limitante. Estas plantas poseen adaptaciones para absorber, retener o impedir la pérdida de agua. Entre estas últimas es posible citar a los géneros Senecio (hojas muy pubescentes), Ephedra (tallos fotosintéticos áfilos) y Parastrephia (hojas muy reducidas, hábito achaparrado). Asimismo, abundan las especies pertenecientes a los géneros Adesmia, Deyeuxia y Fabiana, ya presentes en el Holoceno Medio temprano. Estos taxones están adaptados a la extrema aridez, propia del Holoceno Medio tardío. Durante el Holoceno Tardío también están presentes los géneros mencionados y se incluyen otras especies xerófitas de los géneros Atriplex (hojas de tamaño reducido con abundantes glándulas de sal y hábito achaparrado), Pycnophyllum (hojas reducidas, dispuestas densamente en las ramas y hábito en cojín), Acantholippia, y Hoffmanseggia. Si bien el clima durante el Holoceno Tardío habría sido semejante al actual, con menor aridez que el Holoceno Medio tardío, se mantienen los mismos taxones que reflejan caracteres morfológicos y anatómicos xerofíticos. Estos resultados refuerzan y apoyan los datos paleoambientales obtenidos por otras vías de análisis (sedimentos, diatomeas, granulometrías, entre otros (Tchilinguirian, 2008; Tchilinguirian \& Olivera, 2005; Tchilinguirian et al., 2008; Tchilinguirian \& Morales, 2013.

Con respecto a las áreas de captación de recursos vegetales, sobre la base de los taxones recuperados, se observa un mayor uso del tolar, seguido por el pajonal y la vega durante toda la secuencia, considerando las especies locales que crecen en un radio de aproximadamente $30 \mathrm{~km}$ a partir del sitio CS1. Es importante aclarar que, en otros sectores de la cueva, se registraron también especies no-locales tales como Chusquea lorentziana Griseb. (Poaceae) utilizada para confeccionar intermediarios para puntas de proyectiles y espinas de Trichocereus pasacana (Web) Britton et Rosee (Cactaceae) que pudieron servir a modo de agujas. La primera crece en la región de las yungas, a menos de $2.000 \mathrm{~m} \mathrm{s.m}$. y a $180 \mathrm{~km}$ de CS1. La segunda se desarrolla en la Pre-Puna entre 2.500-3.000 m s. m. a ca. $100 \mathrm{~km}$ del sitio (Pintar \& Rodríguez, 2015).

Para discutir los resultados obtenidos, es interesante considerar el modelo de poblamiento que propone Borrero (1994- 1995) y que incluye tres fases: exploración, colonización y ocupación efectiva del espacio. El modelo se basa en la duración de las ocupaciones y el uso de materias primas. Las dos primeras fases habrían sido procesos lentos, discontinuos y de relleno de espacios vacíos. La exploración inicial corresponde a la primera dispersión de individuos o grupos siguiendo caminos naturales en espacios no habitados. La siguiente es la fase de la colonización que se refiere a la presencia de grupos con áreas de acción específicas que habitan nuevamente sitios ubicados en lugares óptimos, lo cual resulta en una alta visibilidad arqueológica. Por último, la ocupación efectiva se vincula con la fase en la que está habitado todo el espacio deseable durante períodos más prolongados (Borrero \& Franco, 1997; Borrero, 1999, 2013; Franco, 2002; Civalero \& Franco, 2003; Pintar et al., 2016).

Teniendo en cuenta estas tres fases de poblamiento, la secuencia de CS1 corresponde a la fase de ocupación efectiva. En las capas 2(8) a 2(1) se recuperaron especies leñosas integrando los fogones, tales como Acantholippia sp. (Verbenaceae), Adesmia sp. (Fabaceae), Ephedra multiflora (Ephedraceae), Fabiana sp. (Solanaceae) y Parastrephia sp. (Asteraceae). Asimismo, es muy abundante Deyeuxia eminens var. fulva en las camadas de gramíneas que conforman los pisos de ocupación del sitio en las capas 1(1) y 1(2). También están presentes algunos taxones comestibles, tales como Hoffmanseggia eremophila (Fabaceae), del cual se recuperaron frutos en las capas 1(1), 2(4) y 2(6) (Tablas 2 y 3). Es importante destacar que en otros estudios se determinó el uso de especies vegetales no-locales, mencionadas más arriba, y locales para confeccionar tecnofacturas, ya que se hallaron nudos elaborados con Cortaderia speciosa y Deyeuxia sp. (Pintar \& Rodríguez, 2015).

Al igual que en el sitio Quebrada Seca 3, ubicado en el pajonal a $c a .4 .000$ m s. m. (Rodríguez, 1998, 1999, 2000), durante la fase de ocupación efectiva de CS1 se habrían ampliado los radios de forrajeo. Actualmente, estos radios ya están bien establecidos en la microrregión de ANS y se considera paralelamente el uso de la materia prima lítica para la cual se registra, además del uso de las fuentes de obsidiana ubicadas 


\section{N. M. Apóstolo et al. - Macrorrestos vegetales en el sitio arqueológico Cueva Salamanca 1}

en Ona y Laguna Cavi, el comienzo de la explotación de tres nuevas fuentes de obsidiana: Purulla-Chascón y Archibarca, ubicadas hacia el Noroeste y el Salar Hombre Muerto, hacia el Norte (Pintar \& Rodríguez, 2015).

En síntesis, los grupos humanos que habitaron CS1 utilizaron más del $40 \%$ de las plantas de la flora actual del área de ANS durante el Holoceno Medio temprano, Medio tardío y Tardío. Esto indica una ocupación efectiva del sitio pues dichos grupos conocían e identificaban las distintas especies vegetales y las usaban recurrentemente. Al mismo tiempo, implica y supone la intención de explotar, por ejemplo, determinados taxones como combustible, así como también ciertas especies como materia prima para confeccionar tecnofacturas y otras como camadas en áreas de descanso (Pintar \& Rodríguez, 2015).

Por último, es importante destacar que Borrero (1994-1995) plantea este modelo sobre la base de la materia prima lítica, sin tener en cuenta las especies vegetales. En los sitios arqueológicos del área de ANS, hay una excelente preservación de los macrorrestos arqueobotánicos debido a la aridez del ambiente. Por lo tanto, se podría considerar la posibilidad de incorporar estos datos como otra fuente de información para futuras investigaciones arqueológicas.

\section{Contribución de los autores}

Todos los autores participaron en el diseño y realización de la investigación y en la redacción del manuscrito. NMA y MFR preparación muestras y análisis morfo-anatómico, análisis e identificación taxonómica. NMA, elaboración de las figuras. EP, excavación, descripción y estudio del sitio arqueológico y recuperación del material arqueobotánico. MFR y EP, interpretación de resultados a partir de un enfoque arqueobotánico y de trabajos previos realizados en el área de estudio.

\section{Agradecimientos}

A Natalia Mufato y Daniela Acosta, estudiantes de la carrera de Licenciatura en Ciencias Biológicas de la Universidad de Luján, por el procesamiento, acondicionamiento y sistematización del material. Al Lic. Fabián Tricarico, técnico del Servicio de
Microscopía Electrónica del Museo de Ciencias Naturales Bernardino Rivadavia. A Maribel Macchione por el asesoramiento en la edición de algunas fotografías de las figuras. Este trabajo ha sido financiado con fondos de subsidios a la investigación otorgados por el Departamento de Ciencias Básicas de la Universidad Nacional de Luján. Las excavaciones de Cueva Salamanca 1 se llevaron a cabo mediante los siguientes subsidios: PID-CONICET 3-046100/88; PIP-CONICET 6398; PIP-CONICET30-54666038-5; PIP-CONICET 0288; UBACYT 20020090200027; Earthwatch, The Center for Field Research y Fulbright 2011-2012 (E. Pintar).

\section{Bibliografía}

BORRERO, L.A. 1994-1995. Arqueología de la Patagonia. Palimpsestos 4: 9-69.

BORRERO, L. A. 1999. The prehistoric exploration and colonization of Fuego-Patagonia. J. World Prehist.13: 321-355. http://dx.doi.org/10.1023/A:1022341730119.

BORRERO, L.A. 2013. Ranked habitats and the process of human colonization of South America. Quat. Int. 306: 1-4. http://dx.doi.org/10.1016/j.quaint.2013.06.013.

BORRERO, L.A. \& N. FRANCO. 1997. Early Patagonian hunter-gatherers: subsistence and technology. $J$. Anthropol. Res. 53: 219-239. http://dx.doi.org/10.1086/jar.53.2.3631277.

CABRERA, A. L. 1976. Regiones Fitogeográficas Argentinas. En W. F. Kugler (ed.), Enciclopedia Argentina de Agricultura y Jardinería, pp. 1-85, $2^{\circ}$ Ed. Editorial Acme, Buenos Aires.

CIVALERO, M.T. \& N. FRANCO. 2003. Early human occupations in western Santa Cruz province, southernmost South America. Quat. Int. 109-110: 77-86.

http://dx.doi.org/10.1016/S1040-6182(02)00204-5.

CUELLO, A. S. 2006. Guía ilustrada de la flora de Antofagasta de la Sierra, Catamarca (Puna Meridional Argentina). Curso de entrenamiento para la obtención del grado de Licenciatura en Ciencias Biológicas. Universidad Nacional de Tucumán.

D'AMBROGIO DE ARGÜESO, A. 1986. Manual de Técnicas en Histología Vegetal. Editorial Hemisferio Sur. Buenos Aires, Argentina.

FERNÁNDEZ, J., V. MARKGRAF, H. PANARELLO, M. ALBERO, F ANGIOLINI, S VALENCIA \& M. ARRIAGA. 1991. Late Pleistocene-early Holocene environment and climates, fauna, and human occupation in the Argentine Altiplano. Geoarchaeology 6: 251- 272.

http://dx.doi.org/10.1002/gea.3340060303. 


\section{Bol. Soc. Argent. Bot. 54 (1) 2019}

FRANCO, N. 2002. Es posible diferenciar los conjuntos líticos atribuidos a la exploración de un espacio de los correspondientes a otras etapas del poblamiento. Werken 3: 119-132.

GROVE, M. 2009. Hunter-gatherer movement patterns: causes and constraints. J. Anthropol. Archaeol. 28: 222-233. http://dx.doi.org/10.1016/j.jaa.2009.01.003.

GROVE, M. 2010. Logistical mobility reduces subsistence risk in hunting economies. J. Archaeol. Sc. 37: 19131921. http://dx.doi.org/10.1016/j.jas.2010.02.017.

HABER, A. 1992. Pastores y pasturas. Recursos forrajeros en Antofagasta de la Sierra (Catamarca) en relación a la ocupación Formativa. Shincal 2: 15-23.

KELLY, R. 2013. The Lifeways of Hunter-Gatherers. The Foraging Spectrum. Cambridge University Press, New York.

LUPO, L. C. \& M. ECHENIQUE. 2001. Reconstrucción arqueopalinológica de los diversos momentos de ocupación del yacimiento Formativo Moralito. Jujuy, Noroeste Argentino. Ameghiniana 8: 125-130.

MARKGRAF, V. 1985. Paleoenvironmental history of the last 10,000 years in Northwestern Argentina. $\mathrm{Zbl}$. Geol. Paläont. 1: 1739-1749.

MARKGRAF, V. 1987.Paleoclimates of the Southern Argentine Andes. Curr. Res. Pleist. 4: 150-157.

MARKGRAF, V. \& P. BRADBURY. 1982. Holocene Climatic History of South America. Striae 11: 40-45.

PINTAR, E. L. 1996. Prehistoric Holocene adaptations to the Salt Puna of Northwest Argentina. Ph. D. Thesis, Dedman College, Southern Methodist University. ProQuest/UMI. (AAT 9628671).

PINTAR, E. L. 2004. Cueva Salamanca 1: ocupaciones altitermales en la puna sur. Relac. Soc. Argent. Antropol. 29: 357-366.

PINTAR, E. \& M. F. RODRÍGUEZ. 2015. Understanding foraging radius and mobility in a high desert. $J$. Archaeol. Sc. 59: $142-158$. http://dx.doi.org/10.1016/j.jas.2015.04.013.

PINTAR, E., JOHNSON, A. \& S. LAMKIN. 2016. Using Binford's frames of reference to model hunter gatherer mobility and group size in the Andean Puna. 81st Annual Meetings of the Society for American Archaeology. Orlando, Florida, EEUU. Abril 2016.

RODRÍGUEZ, M. F. 1998. Arqueobotánica de Quebrada Seca 3: Recursos vegetales utilizados por cazadoresrecolectores durante el periodo Arcaico en la Puna Meridional Argentina. Tesis Doctoral. Universidad de Buenos Aires, Facultad de Ciencias Exactas y Naturales.

RODRÍGUEZ, M. F. 1999. Arqueobotánica de Quebrada Seca 3 (Puna Meridional Argentina): Especies vegetales utilizadas en la confección de artefactos durante el Arcaico. Relac. Soc. Argent. Antropol. 24: 159-184.
RODRÍGUEZ, M. F. 2000. Woody plant species used during the Archaic period in the Southern Argentine Puna. Archaeobotany of Quebrada Seca 3. J. Archaeol. Sc. 27: 341 - 361 . http://dx.doi.org/10.1006/jasc.1999.0515.

RODRÍGUEZ, M. F. 2004a. Cambios en el uso de los recursos vegetales durante el Holoceno en la Puna meridional argentina. Chungara Rev. Antropol. Chil. (Volumen especial): 403-413.

RODRÍGUEZ, M. F. 2004b. Woody plant resources in the Southern Argentine Puna. Punta de la Peña 9 archaeological site. J. Archaeol. Sc. 31: 1361-1372. http://dx.doi.org/10.1016/j.jas.2004.02.014.

RODRÍGUEZ, M. F. 2005. Human evidence during Middle Holocene in the Salty Argentine Puna. Archaebotanical record analyzes. Quat. Int. 132: 15-22. http://dx.doi. org/10.1016/j.quaint.2004.07.011.

RODRÍGUEZ, M. F. 2013. Acerca de la flora de Antofagasta de la Sierra, Catamarca, Argentina. Ambiente y Paleoambiente. Darwiniana (n. s.) 1: 295-323. https://dx.doi.org/10.14522/darwiniana.2013.12.529.

RODRÍGUEZ, M. F. 2014. Archaeobotany and vegetable resources. Settlement systems and mobility in the Southern Argentinean Puna. In: E. Pintar (ed.), Hunter-gatherers from a high altitude desert. People of the Salt Puna, pp. 145-168. British archaeological reports (BAR). Oxford: Archaeopress, John \& Erica Hedges.

RODRÍGUEZ, M. F. \& Z. E. RÚGOLO DE AGRASAR. 1999. Deyeuxia eminens (Poaceae: Agrostideae) en un sitio arqueológico de la Puna Meridional Argentina (Provincia de Catamarca). Darwiniana 37: 229-242.

RODRÍGUEZ, M. F., Z. RÚGOLO DE AGRASAR \& C. ASCHERO. 2003. El género Deyeuxia (Poaceae, Agrostideae) en sitios arqueológicos de la Puna meridional argentina, Provincia de Catamarca. Chungara Rev. Antropol. Chil. 35: 51-72.

RODRÍGUEZ, M. F. \& C. A. ASCHERO. 2005. Acrocomia chunta (Arecaceae): Raw material for cord making in the Argentinean. Puna. J. Archaeol. Sc. 32: 15341542. http://dx.doi.org/10.1016/j.jas.2005.04.016.

RODRÍGUEZ, M. F.Z. E., RÚGOLO DE AGRASAR, \& C. A. ASCHERO. 2006. El uso de las plantas en unidades domésticas del sitio arqueológico Punta de la Peña 4, Puna meridional argentina. Chungara Rev. Antropol. Chil. 2: 257- 271.

TCHILINGUIRIAN, P. 2008. Paleoambientes Holocenos en la Puna Austral, Provincia de Catamarca: Implicancias geoarqueológicas. Tesis Doctoral. UBA, Facultad de Ciencias Exactas y Naturales. Buenos Aires, Argentina.

TCHILINGUIRIAN, P. \& D. OLIVERA. 2005 Evolución paleoambiental e implicancias geoarqueológicas en Laguna Colorada, Puna Catamarqueña, Argentina. XVI Congreso Geológico Nacional. Actas 4: 261-268. La Plata, 20-23 de septiembre de 2005. 


\section{N. M. Apóstolo et al. - Macrorrestos vegetales en el sitio arqueológico Cueva Salamanca 1}

TCHILINGUIRIAN, P., D. OLIVERA \& L. GRANA. 2008. Expansiones y retrocesos de humedales de altura durante el Holoceno, Puna austral, Argentina. Implicancias ambientales. XVII Congreso Geológico Argentino, Actas 1: 729-730. San Salvador de Jujuy, 7 al 10 de octubre de 2008.

TROLL, C. 1958. Las culturas superiores andinas y el medio geográfico. Rev. Inst. Geog. 5: 3-55.
YACOBACCIO, H. D. 2013.Towards a human ecology for the Middle Holocene in the Southern Puna. Quat. Int. 307: 24-30. http://dx.doi.org/10.1016/j.quaint.2012.08.2109.

ZARLAVSKY, G. E. 2014. Histología Vegetal: Técnicas simples y complejas. Editorial Sociedad Argentina de Botánica. Buenos Aires, Argentina. 
